\title{
ON CYCLIC TRIGONAL RIEMANN SURFACES. I
}

\author{
BY
}

ROBERT D. M. ACCOLA

\begin{abstract}
.
DEFINITION. Call the Riemann surfaces for the equation $y^{3}=P(x)$ cyclic trigonal. For one case of genus 4 ( 2 distinct $g_{3}^{1}$ 's) and all genera greater than 4 , cyclic trigonal Riemann surfaces are characterized by the vanishing properties of the theta function at certain (1/6)-periods of the Jacobian. Also for trigonal Riemann surfaces of genera 5, 6, and 7, homogeneous theta relations are derived using the fact that Prym varieties for trigonal Riemann surfaces are Jacobians.
\end{abstract}

1. Introduction. A closed Riemann surface $W$ which can be realized as a threesheeted covering of the Riemann sphere $\mathbf{P}_{1}$ is called trigonal. If there is an automorphism of period three on $W$ which permutes the sheets of the covering, we shall call $W$ cyclic trigonal. This latter case is equivalent to the condition that all the branch points of the three-sheeted covering have multiplicity three and $W$ is the Riemann surface for the equation

$$
y^{3}=\prod_{i=1}^{s}\left(x-\alpha_{i}\right) \prod_{j=1}^{t}\left(x-\beta_{j}\right)^{2}
$$

where the $s+t$ complex numbers $\alpha_{i}, \beta_{j}$ are distinct and finite. We assume $s+2 t \equiv 0$ (mod 3) so there is no branching over $\infty$ in $\mathbf{P}_{1}$. The genus of $W$ is $p=s+t-2$.

In this paper we derive necessary and sufficient conditions for a Riemann surface of genus $p$ to be cyclic trigonal for $p \geqslant 5$ and for one case of genus 4 . The case $p=2$ is done in [2, p. 94]. The cases $p=3$ and the second case of $p=4$ are open.

The characterization will be in terms of vanishings of the associated theta function at (1/6)-periods of the Jacobian of $W, J(W)$. This will extend to trigonal cyclic Riemann surfaces an analysis analogous to the classical theory of hyperelliptic theta functions when hyperellipticity is characterized by certain vanishings of the theta function at half periods of $J(W)[11]$.

By Torelli's theorem the conformal type of a Riemann surface is determined by the symplectic equivalence class of its period matrices. These results for hyperelliptic and cyclic trigonal Riemann surfaces can be viewed as part of a program of extracting from a period matric (in this case via the theta function) information about special properties a Riemann surface might possess.

Received by the editors December 28, 1981.

1980 Mathematics Subject Classification. Primary 30F10; Secondary 14H15.

Key words and phrases. Riemann surfaces, automorphisms, algebraic curve, Jacobian, Prym variety.

'Research supported by the National Science Foundation. 
Part II of this paper will be devoted to deriving the equation $((1.1))$ of the cyclic trigonal Riemann surface from functions of theta constants, again extending to these surfaces an analysis similar to the hyperelliptic case $[8$, p. 326; 16].

In $\$ 2$ we introduce notation, mention some known results, and prove some preliminary lemmas.

In $\$ 3$ we derive the high order vanishing properties of the cyclic trigonal theta function.

In $\$ 4$ we show that these vanishing properties characterize the existence of a trigonal cyclic covering for $p \geqslant 5$. This analysis appears to be of necessity quite a bit more involved than that of the hyperelliptic case. One must first show the existence of a $g_{3}^{1}$ and then show that the three-sheeted covering of $\mathbf{P}_{1}$ is cyclic. Just as one must divide the hyperelliptic analysis into cases of even and odd genus, so one divides the present analysis according to the residue modulo three of the genus. Each of the three cases presents slightly different problems, and in each case the lower genera present additional problems. Hence the length of this paper.

In $\S 5$ we deal with the one case of $p=4$ that we are able to handle.

Finally, in $\$ 6$ we include some additional remarks about trigonal Riemann surfaces of genus 5, 6, and 7. Here the idea is to define the trigonal locus in Teichmüller space by equations in thetanulls. This section can be read independently of the rest of the paper.

2. Definitions, notation, and preliminary results. In this section we assume the reader is already familiar with the subject of Riemann surfaces. Mainly we will consider those points where notation, definitions, and results are not standard.

$W_{p}$ will stand for a closed Riemann surface of genus $p$. Let $A\left(W_{p}\right)$ denote the full group of automorphisms (conformal self-maps) of $W_{p}$. If $G$ is a finite group of automorphisms, the space of orbits, denoted $W_{p} / G$, is naturally a Riemann surface. The map $W_{p} \rightarrow W_{p} / G$, which takes a point of $W_{p}$ into its $G$-orbit, is an $n$-sheeted covering of closed Riemann surfaces, where $n$ is the order of $G$. Such a covering will be called normal.

An arbitrary $n$-sheeted covering $W_{p} \rightarrow W_{q}$ will be called strongly branched if $p>n^{2} q+(n-1)^{2}[\mathbf{1}]$. In this case, if $n$ is prime, there is only one such covering for given $n$ and $q$.

If $W_{p} \rightarrow W_{q}$ is $n$-sheeted and $W_{p} \rightarrow W_{q^{\prime}}$ is $n^{\prime}$-sheeted then the inequality of Castelnuovo and Severi says that $p \leqslant n q+n^{\prime} q^{\prime}+(n-1)\left(n^{\prime}-1\right)$ under suitable conditions. For our purposes we note that the inequality holds if $\left(n, n^{\prime}\right)=1$ or if $n=n^{\prime}$ is a prime number. Some consequences are stated in the following lemma.

Lemma 2.1. Suppose $W_{p} \rightarrow W_{q}$ is an n-sheeted covering.

(i) If $n$ is prime and $p>2 n q+(n-1)^{2}$ then there is only one such cover with the given $n$ and $q$.

(ii) If $W_{p} \rightarrow W_{q^{\prime}}$ is an $n^{\prime}$-sheeted covering where $q^{\prime}=0, n^{\prime}=3$ and $n=2$ then $p \leqslant 2 q+2$.

(iii) If $n=2$ and $p>4 q+1$ then there is a unique automorphism $T$ of period two so that the genus of $W /\langle T\rangle$ is $q$. 
REMARK. In the light of Lemma 2.1(i) a better definition of strongly branched might be $p<2 n q+(n-1)^{2}$. But with this definition all the results of [1] do not appear to follow.

If $W_{p} \rightarrow W_{q}$ is an $n$-sheeted cover where $n$ is prime and $p>2 n q+(n-1)^{2}$, let $N$ be the normal subgroup of $A\left(W_{p}\right)$ which leaves the fibers of the cover fixed. Then $A\left(W_{p}\right) / N$ is isomorphic to a finite subgroup of $A\left(W_{q}\right)$. If $G \subset A\left(W_{p}\right)$ and $G \cap N=$ $\langle e\rangle$, then we say that $G$ descends to $W_{q}$. That means that $G$ permutes the fibers of the map $W_{p} \rightarrow W_{q}$ and induces a group of automorphisms on $W_{q}$ isomorphic to $G$.

A divisor $D$ on $W_{p}$ is a formal finite sum $\Sigma n_{i} z_{i}$ where $z_{i} \in W_{p}$ and $n_{i} \in Z$. The degree of $D, \operatorname{deg} D$, is $\sum n_{i}$. $D$ is called integral if $n_{i} \geqslant 0$ for all $i$. If $\phi: W_{p} \rightarrow W_{q}$ is an $n$-sheeted cover and $D=\sum n_{i} z_{i}$ is a divisor on $W_{q}$, we call $\sum n_{i} \phi^{-1}\left(z_{i}\right)$ the lift of $D$. The branch points in $\phi^{-1}\left(z_{i}\right)$ are counted according to multiplicity so deg $\phi^{-1}\left(z_{i}\right)=n$ always. If $D=\sum n_{i} z_{i}$ is a divisor on $W_{p}$ we call $\sum n_{i} \phi\left(z_{i}\right)$ the drop of $D$ to $W_{q}$. Thus if $D$ is a divisor on $W_{q}$ the drop of the lift of $D$ is $n D$.

If $D$ is (linearly) equivalent to an integral divisor then $|D|$ will denote the complete linear series of integral divisors equivalent to $D$, denoted $g_{n}^{r}$, where $n=\operatorname{deg} D$ and $r$ is the projective dimension. (We will frequently abuse notation by writing $D \equiv g_{n}^{r}$ for $|D|=g_{n}^{r}$.) We will denote the canonical series $g_{(2 p-2)}^{p-1}$ by $K$ when convenient. If $g_{n}^{r}+g_{n^{\prime}}^{r^{\prime}} \equiv K$ we say one of these linear series is the complement of the other. For complete linear series $g_{n}^{r}$ the Riemann-Roch theorem says $r=n-p+i$, where $i$ is the index of speciality. If $g_{n}^{r}$ is special $(i>0)$ then Clifford's theorem says that $n-2 r \geqslant 0$.

Linear series may be complete or incomplete, may or may not have fixed points, and may be simple or composite. We shall discuss the latter two concepts a little more fully. A $g_{n}^{r}, r \geqslant 2$, will be called simple if the map of $W_{p}$ into $\mathbf{P}_{r}$ (projective space of dimension $r$ ) is one-to-one in general. If $g_{n}^{r}$ is not simple it is called composite. This means that there is a cover $\phi: W_{p} \rightarrow W_{q}$ of $t$ sheets, $t \geqslant 2$, and a simple linear series $g_{(n-\varepsilon) / t}^{r}$ on $W_{q}$, the divisors of which lift to the nonfixed points of $g_{n}^{r}$. $\varepsilon$ is the number of fixed points of $g_{n}^{r}$. If $g_{n}^{r}$ is complete, so is $g_{(n-\varepsilon) / t}^{r}$. The set of fibers of the map $\phi$ will be called an involution and denoted $\gamma_{t}^{1}$. We say that $g_{n}^{r}$ is compounded of the involution $\gamma_{t}^{1}$. If $q=0$ then $\gamma_{t}^{1}=g_{t}^{1}$.

A linear series $g_{p-1}^{r}$ such that $2 g_{p-1}^{r} \equiv K$ will be called half-canonical and be abbreviated (1/2)-K. If $2 n g_{p-1}^{r} \equiv n K$ we say $g_{n}^{r}$ is $(1 / 2 n)-n K$. We will be particularly concerned with (1/6)-3K linear series which includes the possibility of $(1 / 2)-K$.

Lemma 2.2 (CASTElnUovo's ineQuality AND theorem). Suppose $W_{p}$ admits a simple $g_{n}^{r}$. Then

$$
p \leqslant \frac{n-r+\varepsilon}{r-1} \frac{n-1-\varepsilon}{2}
$$

where $\varepsilon=0,1,2, \ldots$, or $r-2$ and $n-r+\varepsilon \equiv 0(\bmod r-1)$. If we have equality in (2.1) then $W_{p}$ admits a $g_{m}^{1}$ which imposes two linear conditions on $g_{n}^{r}[5]$.

The main use we will make of the second part of Lemma 2.2 is the following. 
LEMMA 2.3. If $W_{3 r+1}$ admits a simple $g_{3 r}^{r}$ which is not (1/2)-K, then $2 g_{3 r}^{r} \equiv g_{6 r}^{3 r-1} Z$ $K$ and we have equality in (2.1). Then $W_{3 r+1}$ admits a $g_{3}^{1}$ or a $g_{4}^{1}$. If $W_{3 r+1}$ admits a $g_{3}^{1}$ and $g_{3 r}^{r}+h_{3 r}^{r} \equiv K$, then one of these two linear series is $r g_{3}^{1}$.

A Riemann surface admitting a $g_{3}^{1}$ without fixed points will be called trigonal. A Riemann surface which is a two-sheeted covering of a surface of genus $q$ will be called $q$-hyperelliptic (abbreviated $(q-\mathrm{H})$ ). Thus, by Lemma $2.1(\mathrm{ii})$, if $W_{p}$ is $(q-\mathrm{H})$ and trigonal then $p \leqslant 2 q+2$. Thus a Riemann surface of genus five cannot be $(1-\mathrm{H})$ (elliptic-hyperellitpic) and trigonal simultaneously.

By the Riemann-Roch theorem it follows that a canonical divisor containing $n-1$ points of a divisor of a $g_{n}^{1}$ must contain the $n$ th. Applying this for $n=3$ yields the following lemma.

LEMMA 2.4 [4]. Suppose $g_{n}^{r}$ and $g_{n^{\prime}}^{r^{\prime}}$ are complementary linear series on a trigonal Riemann surface. Then one of these is compounded of the $g_{3}^{1}$. A (1/2)-K linear series on a trigonal Riemann surface is compounded of the $g_{3}^{1}$.

If all the branch points of the covering $W_{p} \rightarrow \mathbf{P}_{1}$ given by a $g_{3}^{1}$ are of multiplicity three then the covering is normal with group $Z_{3}$. In this case $W_{p}$ will be said to be cyclic trigonal. (This is one of the few cases where the multiplicity of the branching alone suffices to insure a normal covering.) We will call a branch point of multiplicity three a 3-branch point. Thus a trigonal Riemann surface of genus $p$ with $p+2$ 3-branch points in the three-sheeted cover of $\mathbf{P}_{1}$ will be cyclic trigonal by the Riemann-Hurwitz formula.

If $p \geqslant 5 W_{p}$ admits at most one $g_{3}^{1}$ without fixed points and a $W_{4}$ admits at most two which are complementary.

Let $J\left(W_{p}\right)$ be the Jacobian of $W_{p}$ [12]. If we fix a basepoint in $W_{p}$ then we have the usual map of $W_{p}$ and of divisors on $W_{p}$ into $J\left(W_{p}\right)$. If we fix a canonical homology basis on $W_{p}$, then we have determined a period matrix $(\pi i E, B)^{p \times 2 p}$ and we can form the theta function $\theta(u ; B)$, where $u \in C^{p}$ and $B$ can be considered a function on, say, Teichmüller space.

We often make a statement that "the theta function" has such and such a property. When we do, we assume a basepoint chosen, if necessary, and a canonical homology basis chosen. Then there is a uniquely defined theta function (with zero theta characteristic). The stated property will, however, be independent of the choices. For instance, for genus three, the following is such a statement: $W_{3}$ is hyperelliptic if and only if the theta function vanishes at a half period to order two.

If the theta function vanishes at a point $F \in J\left(W_{p}\right)$ to order $k$, we will often say that $\theta(F)=0 k$ times. This is well defined since the theta divisor is well defined on $J\left(W_{p}\right)$.

Basic to all this analysis is Riemann's theorem [13], parts of which are collected in the following theorem.

THEOREM 2.5. Suppose a basepoint and a canonical homology basis have been chosen for $W_{p}$ and the map $u: W_{p} \rightarrow J\left(W_{p}\right)$ is defined. Then there is a point $\kappa$ in $J\left(W_{p}\right)$ so that:

(a) For any $F \in J(W)$ there is a divisor $D$ of degree $p$ on $W_{p}$ so that

$$
u(D)+\kappa \equiv F \text {. }
$$


(b) $i(D)=0$ if and only if $\theta(F) \neq 0$, in which case $D$ is the unique solution to (2.2).

(c) If $\theta(F)=0 r+1$ times then (2.2) can be solved by divisors $D$ such that $|D|=g_{p-1}^{r}$. Such a $g_{p-1}^{r}$ will be said to correspond to $F$.

(d) If $2 n F \equiv 0$ in part (c) then $g_{p-1}^{r}$ is $(1 / 2 n)-n K$.

(e) If $\theta(F)=\theta(-F)=0, u(D)+\kappa \equiv F$ and $u\left(D^{\prime}\right)+\kappa \equiv-F$, then $D+D^{\prime} \equiv K$.

(f) $u(K)+2 \kappa \equiv 0$.

Now we apply Riemann's theorem to smooth abelian covers [2]. Suppose $\phi$ : $W_{p} \rightarrow W_{q}$ is an $n$-sheeted unbranched normal covering where the Galois group $G$ is abelian. Let $a$ denote the map that lifts divisors of degree zero from $W_{q}$ to $W_{p}$. Suppose basepoints $z, z_{0}$ chosen so that $\phi(z)=z_{0}$. Then there is an induced map, also denoted $a, a: J\left(W_{q}\right) \rightarrow J\left(W_{p}\right)$ so that the following diagram commutes [2, p. 4] $($ (용 0 denotes divisors of degree zero):

$$
\begin{array}{ccc}
\mathscr{Q}_{0}\left(W_{p}\right) & \stackrel{u}{\rightarrow} & J\left(W_{p}\right) \\
\uparrow a & & \uparrow a \\
\mathscr{Q}_{0}\left(W_{q}\right) & \stackrel{u_{0}}{\rightarrow} & J\left(W_{q}\right)
\end{array}
$$

Then ker $a\left(\subset J\left(W_{q}\right)\right)$ is isomorphic to $G$. Moreover, to every finite subgroup $A$ of $J\left(W_{q}\right)$ there is a suitable smooth abelian cover of $W_{q}$ so that the diagram commutes. In this case we will say that the cover $W_{p} \rightarrow W_{q}$ corresponds to the finite subgroup $A$.

We need to know more about this situation.

LEMMA 2.6. In the immediately preceding situation, suppose there is a point $E \in J\left(W_{q}\right)$ so that the theta function vanishes at the points of the coset $E+\operatorname{ker} a$ to orders $r_{1}+1, r_{2}+1, \ldots, r_{n}+1$. Then the theta function for $J\left(W_{p}\right)$ vanishes at aE to order $n+\sum r_{i}$. Moreover, if $r_{i} \geqslant 0$ for $i=1, \ldots, n$ and one of the linear series $g_{q-1}^{r_{i}}$ on $W_{q}$ corresponding to a period in $E+\operatorname{ker} a$ is simple, then the $g_{p-1}^{\Sigma r_{i}+n-1}$ on $W_{p}$ corresponding to aE in $J\left(W_{p}\right)$ is also simple.

Proof [2, p. 74]. The last statement is not proven in the reference. It follows from familiar procedures.

We need to quote another theorem from [2, p. 77].

Lemma 2.7. Suppose $W_{p} \in(q-\mathrm{H})$. This property is characterized by the vanishing properties of the theta function at certain half-periods if:

when $p$ is odd $6 q \leqslant p+3$,

when $p$ is even $6 q \leqslant p$.

If $W_{p}$ is trigonal $(p \geqslant 5)$ and also $(q-\mathrm{H})$ then we know $p \leqslant 2 q+2$ (Lemma 2.1(ii)). Consequently $p$ and $q$ cannot be related as in Lemma 2.7. Thus $W_{p}$ cannot have the vanishing properties which would insure that $W_{p} \in(q-\mathrm{H})$ for $q$ in the ranges of Lemma 2.7. We shall refer to this as the nonvanishing properties of a trigonal Riemann surface.

We conclude this section with some more results on a $W_{3 r+1}$ admitting a $g_{3 r}^{r}$ (in addition to Lemma 2.3). The following lemma will be used to exclude the possibility of a $g_{4}^{1}$ in applying Lemma 2.3 later in the paper. 
LEMMA 2.8. Suppose $p=3 r+1$ and $W_{p}$ admits a fixed point free automorphism $T$ of period three. Then $W_{p}$ cannot admit a unique $g_{4}^{1}$.

Proof. Suppose $W_{p}$ admits a unique $g_{4}^{1}$. Then $T$ permutes the divisors of $g_{4}^{1}$. Since $g_{4}^{1}$ is parametrized by $\mathbf{P}_{1}$, there is at least one divisor $D_{0}$ in $g_{4}^{1}$ so that $T D_{0}=D_{0}$. Thus $T$ permutes the points of $D_{0}$, preserving multiplicities. We conclude that $T$ has a fixed point among the points of $D_{0}$ since $(3,4)=1$, contradiction. Q.E.D.

LEMMA 2.9. Suppose $W_{3 r+1}$ admits a simple (1/2)- $K g_{3 r}^{r}$, where $r \geqslant 10$. Thus $W_{3 r+1}$ admits a $g_{3}^{1}, a g_{4}^{1}$, or a $\gamma_{3}^{1}$ which is the set of fibers of a three-sheeted cover of a torus $W_{1}$ [7]. In the last case $g_{3 r}^{r}=\left|g_{3 r}^{r-1}\right|$, where $g_{3 r}^{r-1}$ is the lift of a $g_{r}^{r-1}$ on $W_{1}$.

REMARK. With the stated hypotheses (which are more restrictive than those of [7]), a $g_{3}^{1}$ is excluded by Lemma 2.4 .

Proof. For the first part of the proof we cite the reference. For the last statement we refer to a generalization of the Riemann-Roch theorem due to Castelnuovo [6, $\mathrm{p}$. 324] to infer that any divisor $D_{1}$ in $\gamma_{3}^{1}$ imposes two conditions on $g_{3 r}^{r}$. But by [3] two divisors in $\gamma_{3}^{1}, D_{1}+D_{2}$, impose at most three conditions on $g_{3 r}^{r}$ since $\left|D_{1}+D_{2}\right|=g_{6}^{1}$ on $W_{3 r+1}$. Thus any $D_{2}$ in $\gamma_{3}^{1}$ imposes one condition on $g_{3 r}^{r}-D_{1}=g_{3 r-3}^{r-2}$. That is, $g_{3 r-3}^{r-2}$ is composite, being compounded of $\gamma_{3}^{1}$. Since $D_{1}+g_{3 r-3}^{r-2}$ is the lift of a $g_{r}^{r-1}$ from $W_{1}$, the result follows. Q.E.D.

LEMma 2.10. Suppose $W_{3 r+1}$ admits a simple (1/2)-K $g_{3 r}^{r}$ and the $\gamma_{3}^{1}$ of Lemma 2.9 . Then there are three additional complete (1/2)-K linear series of dimension $r-1$.

PROOF. There is a $g_{r}^{r-1}$ on $W_{1}$ whose lift when completed is $g_{3 r}^{r}$. On $W_{1}$ there are three other linear series, $h_{r}^{r-1}, k_{r}^{r-1}, l_{r}^{r-1}$, so that their doubles are linearly equivalent to $2 g_{r}^{r-1}$. When lifted, the three linear series become half-canonical since the lift of $g_{r}^{r-1}$ is. If the lift of $h_{r}^{r-1}$ is denoted $h_{3 r}^{r-1}$, we see it is complete since $g_{3 r}^{r}$ is the unique linear series of that dimension and degree. Q.E.D.

REMARK. It also follows that the sum of the four $(1 / 2)-K$ linear series on $W_{p}$ is bicanonical.

LEMMA 2.11. Suppose $W_{3 r+1}, r \geqslant 2$, admits a $g_{3}^{1}$ without fixed points, and $r g_{3}^{1}$ is the unique $(1 / 2)-K g_{3 r}^{r}$. Then there does not exist a complete $(1 / 2)-K h_{3 r}^{r-1}$.

Proof. Suppose there does exist a complete $(1 / 2)-K h_{3 r}^{r-1}$. Then $h_{3 r}^{r-1} \equiv(r-1) g_{3}^{1}$ $+x+y+z$, where $x, y$, and $z$ are three points on $W_{3 r+1}$ (Lemma 2.4). Since $2 h_{3 r}^{r-1} \equiv 2 g_{3 r}^{r} \equiv 2 r g_{3}^{1}$, we see that $2 x+2 y+2 z \equiv 2 g_{3}^{1} \equiv g_{6}^{2}$, which is necessarily complete and composite. Then $2 x+2 y+2 z=D_{1}+D_{2}$ when $D_{1}$ and $D_{2}$ are divisors in $g_{3}^{1}$. Thus two of the points, say $x+y$, must be in $D_{1}$. It follows that we can assume $D_{1}=2 x+y$ and $D_{2}=y+2 z$. Therefore $D_{1}=D_{2}, x=z$ and $x+y+$ $z=2 x+y=D_{1}$. Thus the dimension of $h_{3 r}^{r-1}$ must be $r$, a contradiction. Q.E.D.

This property proven in Lemma 2.11 will also be referred to as a nonvanishing property for the theta function for such a trigonal Riemann surface. For on the Jacobian the theta function does not vanish to order $r$ at any half-period. 
3. Vanishing properties of the cyclic trigonal theta functions. Let $W_{p}$ be the Riemann surface for the equation

$$
y^{3}=\prod_{i=1}^{s}\left(x-\alpha_{i}\right) \prod_{j=1}^{t}\left(x-\beta_{j}\right)^{2}
$$

where the $s+t$ complex numbers $\alpha_{i}, \beta_{j}$ are distinct and finite. Without loss of generality we assume $s+2 t \equiv 0(\bmod 3)$ and $s \leqslant t$. Consequently, the three-sheeted covering is unbranched over $\infty$ and $p=s+t-2$. On $W_{p} y$ is a single valued function whose divisor can be written

$$
(y)=\sum_{i=1}^{s} a_{i}+\sum_{j=1}^{t} 2 b_{j}-\left(\frac{s+2 t}{3}\right)\left(\infty_{1}+\infty_{2}+\infty_{3}\right),
$$

where $a_{i}$ (respectively $b_{j}$ ) is the point on $W_{p}$ over $\alpha_{i}\left(\right.$ respectively $\beta_{j}$ ) and $\infty_{1}+\infty_{2}+$ $\infty_{3}$ is the divisor of three distinct points over $\infty$. (When convenient we shall denote $a_{i}$ by $c_{i}$ for $i=1,2, \ldots, s$ and $b_{j}$ by $c_{j+s}$ for $j=1,2, \ldots, t$.)

A canonical divisor $K$ on $W_{p}$ can be written

$$
K=2 \sum_{i=1}^{s} a_{i}+2 \sum_{j=1}^{t} b_{j}-2\left(\infty_{1}+\infty_{2}+\infty_{3}\right)
$$

Writing $g_{3}^{1}$ for $\left|\infty_{1}+\infty_{2}+\infty_{3}\right|$ and using the fact that $\Sigma a_{i}+2 \Sigma b_{j} \equiv((s+2 t) / 3) g_{3}^{1}$, we see that

$$
\begin{aligned}
K & \equiv 2 \Sigma a_{i}-\Sigma a_{i}+\left(\left(\frac{s+2 t}{3}\right)-2\right) g_{3}^{1} \\
& \equiv\left(\frac{2 t-2 s-6}{3}\right) g_{3}^{1}+s g_{3}^{1}+\Sigma a_{i} \\
& \equiv 2\left(\left(\frac{t-s-3}{3}\right) g_{3}^{1}+2 \Sigma a_{i}\right)
\end{aligned}
$$

since $g_{3}^{1}=\left|3 a_{i}\right|$ for any $i$. If $t>s$ let $r_{0}=(t-s-3) / 3$ or $t-s=3\left(r_{0}+1\right)$. Thus for $t>s$ the following is half-canonical:

$$
g_{p-1}^{r_{0}}=r_{0} g_{3}^{1}+2 \sum_{i=1}^{s} a_{i}
$$

We shall see shortly why it has dimension precisely $r_{0}$.

Let $z_{0}$ in $W_{p}$ be a basepoint for the map $u: W_{p} \rightarrow J\left(W_{p}\right)$ and let $\left|z_{0}+z_{1}+z_{2}\right|=g_{3}^{1}$. ( $z_{0}$ may or may not be an $a_{i}$ or $b_{j}$.) Now pick a point $G_{0}$ in $J\left(W_{p}\right)$ so that $3 G_{0} \equiv u\left(g_{3}^{1}\right)$. The choices of $z_{0}$ and $G_{0}$ are made one and for all. The results of this paper, however, will not depend on these choices.

Now $\left|3 c_{k}\right|=g_{3}^{1}, k=1,2, \ldots, s+t$, so there are points $C_{k}$ in $J\left(W_{p}\right)$ satisfying the equation

$$
u\left(c_{k}\right)=C_{k}+G_{0} \quad\left(3 C_{k} \equiv 0\right) .
$$


(Write $A_{i}=C_{i}$ for $i=1, \ldots, s$ and $B_{j}=C_{s+j}$ for $j=1,2, \ldots, t$.) On $W_{p} K \equiv 2 \Sigma c_{k}-$ $2 g_{3}^{1}$, so in $J\left(W_{p}\right)$ we have

$$
\begin{aligned}
-2 \kappa & =u(K)=2 \Sigma u\left(c_{k}\right)-2 u\left(g_{3}^{1}\right) \\
& =2 \Sigma C_{k}+2(s+t-3) G_{0}=2 \Sigma C_{k}+2(p-1) G_{0}
\end{aligned}
$$

Thus $\kappa=-\Sigma C_{k}-(p-1) G_{0}+E_{2}$, where $2 E_{2} \equiv 0$ in $J\left(W_{p}\right)$.

Definition. $E_{1}=E_{2}+\sum_{k=1}^{s+t} C_{k}$ in $J\left(W_{p}\right)$.

Consequently, $6 E_{1} \equiv 0, E_{1}+E_{2}=\Sigma C_{k}=\Sigma A_{i}+\Sigma B_{j}$ and $\kappa=-E_{1}-(p-1) G_{0}$. Thus if $\varepsilon_{i} \geqslant 0, \sum \varepsilon_{i}=p-1$ then

$$
u\left(\sum \varepsilon_{i} c_{i}\right)+\kappa=-\left(E_{1}-\sum \varepsilon_{i} C_{i}\right) .
$$

LeMma 3.1. $2 E_{1} \equiv 0$ if and only if $\sum_{k=1}^{s+t} C_{k} \equiv 0$.

This follows directly from the definition. By (3.2) and (3.5) we have

$$
\Sigma A_{i}+2 \Sigma B_{j}+(s+2 t) G_{0}-(s+2 t) G_{0} \equiv 0,
$$

so

$$
\Sigma A_{i} \equiv \Sigma B_{j}, \quad E_{1}+E_{2} \equiv 2 \Sigma A_{i} \equiv 2 \Sigma B_{j},
$$

and

$$
2 E_{1} \equiv \Sigma A_{i} \equiv \Sigma B_{j} .
$$

It now follows from (3.4) that if $r_{0} \geqslant 0$,

$$
u\left(g_{p-1}^{r_{0}}\right)+\kappa \equiv E_{2} .
$$

Lemma 3.2. Suppose $\sum_{i=1}^{s} \varepsilon_{i} A_{i}+\sum_{j=1}^{t} \delta_{j} B_{j} \equiv 0$, where

(i) $\varepsilon_{i}, \delta_{j}=0,1$, or 2 ,

(ii) $\Sigma \varepsilon_{i}+\Sigma \delta_{j} \equiv 0(\bmod 3)$.

Then only one of the following three cases holds.

(i) $\varepsilon_{1}=\cdots=\varepsilon_{s}=\delta_{1}=\cdots=\delta_{t}=0$,

(ii) $\varepsilon_{1}=\cdots=\varepsilon_{s}=1 ; \delta_{1}=\cdots=\delta_{t}=2$,

(iii) $\varepsilon_{1}=\cdots=\varepsilon_{s}=2 ; \delta_{1}=\cdots=\delta_{t}=1$.

Proof. Suppose $\Sigma \varepsilon_{i}+\Sigma \delta_{j}=3 \gamma$. Then

$$
\sum \varepsilon_{i}\left(u\left(a_{i}\right)-G_{0}\right)+\Sigma \delta_{j}\left(u\left(b_{j}\right)-G_{0}\right) \equiv 0
$$

or

$$
\sum \varepsilon_{i} u\left(a_{i}\right)+\Sigma \delta_{j} u\left(b_{j}\right) \equiv\left(\sum \varepsilon_{i}+\Sigma \delta_{j}\right) G_{0} \equiv \gamma u\left(g_{3}^{1}\right) .
$$

Therefore, there is a function $z$ on $W_{p}$ whose divisor is

$$
\sum \varepsilon_{i} a_{i}+\sum \delta_{j} b_{j}-\gamma\left(\infty_{1}+\infty_{2}+\infty_{3}\right)
$$

and

$$
z^{3}=\Pi\left(x-\alpha_{i}\right)^{\varepsilon_{i}} \Pi\left(x-\beta_{j}\right)^{\delta_{i}} .
$$

If $p \geqslant 5, g_{3}^{1}$ is unique and the result follows. For general $p$ the result follows by observing that the $a_{i}$ 's and $b_{j}$ 's are fixed points for a unique automorphism of period three since the stabilizer of any point in the full group of automorphisms on a Riemann surface is cyclic. Q.E.D. 
Referring to (3.4), an argument analogous to the above shows that no subsum of $2 \Sigma a_{i}$ is linearly equivalent to $g_{3}^{1}$. By Lemma 2.4 if follows that the half-canonical linear series $g_{p-1}^{r_{0}}$ must be complete.

We now consider (1/6)-periods in $J\left(W_{p}\right)$ of the type

$$
E_{1}-\sum_{i=1}^{s} \varepsilon_{i} A_{i}-\sum_{j=1}^{t} \delta_{j} B_{j}
$$

where $\varepsilon_{j}, \delta_{j}=0,1$, or 2 and $\sum \varepsilon_{i}+\Sigma \delta_{j} \equiv s+t(\bmod 3)$.

First we count that there are $3^{p}$ such periods $(p=s+t-2)$. For there are $3^{p+1}$ ways of writing periods in the form (3.9). The relation $\sum A_{i} \equiv \sum B_{j}$, and the fact that there are essentially no other relations of this type (Lemma 3.2), means that there are actually $3^{p}$ distinct periods of type (3.9). Notice that three times any period of type (3.9) is the half-period $E_{2}$. Also $3 E_{1} \equiv E_{2}$. If we subtract $E_{2}=E_{1}-\sum_{k=1}^{s+t} C_{k}$ from all of the $3^{p}$ periods of type (3.9) we obtain a group of $3^{p}(1 / 3)$-periods which we shall call $\mathcal{G}$. Thus the (1/6)-periods of type (3.9) are the coset $E_{2}+\mathcal{G}$ in $J\left(W_{p}\right)$, a coset closed under taking negatives.

To derive the vanishing properties of the theta function for $W_{p}$ we refer to [2, Corollary 3 , p. 30]. This corollary tells us the vanishing, or possibly nonvanishing, of the theta function at (1/6)-periods of type (3.9). (For an integer $n$, let $\bar{n}$ be the smallest nonnegative residue of $n$ modulo 3.) Let

(3.10) (i) $\sum \varepsilon_{i}+\Sigma \delta_{j}=s+t-3 \tau_{0}$,

(3.10) (ii) $\Sigma\left(\overline{\left.\varepsilon_{i}+1\right)}+\sum\left(\overline{\delta_{j}+2}\right)=s+t-3 \tau_{1}\right.$,

(3.10)(iii) $\sum\left(\overline{\varepsilon_{i}+2}\right)+\sum\left(\overline{\delta_{j}+1}\right)=s+t-3 \tau_{2}$.

Then the theta function vanishes at the indicated (1/6)-period to order $\sum_{i=0}^{2} \max \left(0, \tau_{i}\right)$. Since $\tau_{0}+\tau_{1}+\tau_{2}=0$, one sees this is simply the maximum of the three numbers $\left|\tau_{0}\right|,\left|\tau_{1}\right|,\left|\tau_{2}\right|$.

Now we shall rewrite the period (3.9) as

$$
E_{1}-\sum_{s_{1}}^{s_{1}} A-\sum_{s_{2}}^{s_{2}} 2 A-\sum_{\pi_{1}}^{t_{1}} B-\sum_{\pi_{2}}^{t_{2}} 2 B
$$

where $\varsigma_{1}$ is the subset of indices in (3.9) where $\varepsilon_{i}=1$, card $\varsigma_{1}=s_{1} ; \S_{2}$ is the subset of indices in (3.9) where $\varepsilon_{i}=2$, card $S_{2}=s_{2} ; \sigma_{1}$ is the subset of indices in (3.9) where $\delta_{j}=1$, card $T_{1}=t_{1} ; T_{2}$ is the subset of indices in (3.9) where $\delta_{j}=2$, card $\mathscr{T}_{2}=t_{2}$. Let $\delta_{0}$ be the subset of indices in (3.9) where $\varepsilon_{i}=0$, card $\varsigma_{0}=s_{0}$, and let $\widetilde{T}_{0}$ be the subset of indices in (3.9) where $\delta_{j}=0$, card $\widetilde{T}_{0}=t_{0}$. Then $s_{0}+s_{1}+s_{2}$ $=s$ and $t_{0}+t_{1}+t_{2}=t$. We rewrite (3.10) in the form

(3.11) (i) $s_{1}+2 s_{2}+t_{1}+2 t_{2}=s+t-3 \tau_{0}$,

(3.11) (ii) $s_{0}+2 s_{1}+t_{2}+2 t_{0}=s+t-3 \tau_{1}$, or$$
\text { (3.11)(iii) } s_{2}+2 s_{0}+t_{0}+2 t_{1}=s+t-3 \tau_{2} \text {, }
$$

(3.12) (i) $3 \tau_{0}=\left(s_{0}-t_{2}\right)-\left(s_{2}-t_{0}\right)=\mu_{0}-\mu_{2}$,

(3.12) (ii) $3 \tau_{1}=\left(s_{2}-t_{0}\right)-\left(s_{1}-t_{1}\right)=\mu_{2}-\mu_{1}$,

(3.12)(iii) $3 \tau_{2}=\left(s_{1}-t_{1}\right)-\left(s_{0}-t_{2}\right)=\mu_{1}-\mu_{0}$, 
where

$$
\mu_{0}=s_{0}-i_{2}, \quad \mu_{1}=s_{1}-t_{1} \text { and } \mu_{2}=s_{2}-t_{0} .
$$

Thus $3 \tau=\max \left|\mu_{i}-\mu_{j}\right|$ where the theta function vanishes at the $(1 / 6)$-period (3.9) to order $\tau$.

From (3.12) we could derive all the vanishing properties of the theta function at periods of type (3.9). For instance, we see that the theta function does not vanish if $\mu_{0}=\mu_{1}=\mu_{2}=(t-s) / 3$. Also $\theta\left(E_{2}\right)=0(t-s) / 3$ times. However, we shall limit ourselves to deriving those high order vanishing properties that will characterize the existence of a cyclic trigonal Riemann surface.

Just as one usefully divides the cases for the hyperelliptic vanishing properties into odd and even genera, so we shall divide the genera according to their residues modulo three. Also the case $p \equiv 1(\bmod 3)$ is further divided into two cases since the existence of a half-canonical $g_{3 r}^{r}(p=3 r+1)$ introduces further complications. Consequently, we distinguish four cases.

Case $1 . p \equiv 1(\bmod 3)$ and $s>0$. Let $p=3 r+1$. In this case the theta function vanishes at the $(1 / 6)$-period $E_{1}$ to order $r+1 . E_{1}$ is not a $(1 / 2)$-period. At $(1 / 6)$-periods of type

$$
E_{1}-\left(C_{k}+C_{1}+C_{m}\right) \text { or } E_{1}-\left(2 C_{k}+C_{l}\right),
$$

the theta function vanishes to order $r$ (except when $s=3$ and $C_{k}+C_{l}+C_{m}=A_{1}+$ $\left.A_{2}+A_{3}\right)$. Since $2 E_{1}=\sum A_{i}=\sum B_{j}$ by (3.7) we see that none of these $(1 / 6)$ periods are $(1 / 2)$-periods. There are two aspects of these high order vanishings that we will consider.

The first is the number of (1/6)-periods of type (3.13). This number is clearly

$$
\left(\begin{array}{c}
p+2 \\
3
\end{array}\right)+2\left(\begin{array}{c}
p+2 \\
2
\end{array}\right) .
$$

However, recall that if $\theta(F)=0$ then $\theta(-F)=0$ also. It is possible for two of the periods of type (3.14) to sum to zero. For example, if $s=6$ then

$$
\left(E_{1}-\left(A_{1}+A_{2}+A_{3}\right)\right)+\left(E_{1}-\left(A_{4}+A_{5}+A_{6}\right)\right)=0
$$

by (3.7). What we really want to count is the number of pairs $\{F,-F\}$ when $\theta(F)=0$ and $F$ is of type (3.13). The following lemma is stated in a form useful for the later characterizations of $\S 4$.

LEMMA 3.3. Let $p=3 r+1, r \geqslant 2$. If $W_{p}$ is cyclic trigonal with $s>0$ then there are more than $\left(\begin{array}{c}p+1 \\ 3\end{array}\right)+2\left(\begin{array}{c}p+1 \\ 2\end{array}\right)$ pairs $\{F,-F\}$ where $F$ is of type (3.13) so that the theta function vanishes to order $r$ at $F$.

Proof. We enumerate all cases in tables. We must account for all (1/6)-periods of type (3.13). In the following tables $F$ stands for a (1/6)-period of type (3.13) except that we also include the vanishings to order $r+1$. If $-F$ is also of type (3.13) it is written in. A blank for $-F$ means it is not of type (3.13). The indices on the $A$ 's and $B$ 's are to be taken as representative of the possibilities. The numbers in the last column are derived by permutations of the indices over the possibilities. For 
example, in the second row of the first table below, where $s=3$ and $t=6$, $E_{1}-\left(A_{1}+A_{2}+B_{1}\right)$ really represents 18 possibilities since there are three choices for the $A$ 's and six choices for the $B$ 's.

Case. $s=3, t=6, p=7$.

Order of vanishing of the theta function

$r+1$
$r$
$r$
$r$
$r$
$r$
$r$

$F$

$$
\begin{aligned}
& E_{1}-\left(A_{1}+A_{2}+A_{3}\right) \\
& E_{1}-\left(A_{1}+A_{2}+B_{1}\right) \\
& E_{1}-\left(A_{1}+B_{1}+B_{2}\right) \\
& E_{1}-\left(B_{1}+B_{2}+B_{3}\right) \\
& E_{1}-\left(2 A_{1}+B_{1}\right) \\
& E_{1}-\left(B_{1}+2 B_{2}\right) \\
& E_{1}-\left(A_{1}+2 A_{2}\right)
\end{aligned}
$$

$-F$

$E_{1}$
$E_{1}-\left(A_{3}+2 B_{1}\right)$

$E_{1}-\left(B_{4}+B_{5}+B_{6}\right)$

$E_{1}-\left(2 A_{2}+A_{3}\right)$ number of pairs $\{F,-F\}$

10

18

30

3

There are 124 pairs $\{F,-F\}$ where $F$ is of type (3.13) and the theta function vanishes to order $r$ at $F .124>112$ where

$$
112=\left(\begin{array}{c}
p+1 \\
3
\end{array}\right)+2\left(\begin{array}{c}
p+1 \\
2
\end{array}\right), \quad p=7 \text {. }
$$

Case. $s=3, t \geqslant 9, p=t+1$.

Order of vanishing of the theta function

$r+1$
$r$
$r$
$r$
$r$
$r$
$r$

F

$E_{1}$

$E_{1}-\left(A_{1}+A_{2}+B_{1}\right)$

$E_{1}-\left(A_{1}+B_{1}+B_{2}\right)$

$E_{1}-\left(B_{1}+B_{2}+B_{3}\right)$

$E_{1}-\left(2 A_{1}+B_{1}\right)$

$E_{1}-\left(2 B_{1}+B_{2}\right)$

$E_{1}-\left(A_{1}+2 A_{2}\right)$
$-F$

$E_{1}-\left(A_{1}+A_{2}+A_{3}\right)$

$E_{1}-\left(A_{3}+2 B_{1}\right)$

(a)

$E_{1}-\left(2 A_{2}+A_{3}\right)$ number of pairs

$\{F,-F\}$

1

$3 t$

$3\left(\begin{array}{l}t \\ 2\end{array}\right)$

$\left(\begin{array}{l}t \\ 3\end{array}\right)$

$3 t$

$t(t-1)$

3

There are

$$
3 t+3\left(\begin{array}{l}
t \\
2
\end{array}\right)+\left(\begin{array}{l}
t \\
3
\end{array}\right)+3 t+t(t-1)+3=\left(\begin{array}{c}
t+3 \\
3
\end{array}\right)+2\left(\begin{array}{c}
t+3 \\
2
\end{array}\right)-3 t-4
$$

pairs $\{F,-F\}$ of type (3.13) where $\theta(F)=0 r$ times. This number is larger than $\left(\begin{array}{c}p+1 \\ 3\end{array}\right)+2\left(\begin{array}{c}p+1 \\ 2\end{array}\right)$.

Case. $s=6, t=6, p=10$.

Order of vanishing of

F the theta function

$r+1$
$r$
$r$
$r$
$r$
$r$

$E_{1}$

$E_{1}-\left(A_{1}+A_{2}+A_{3}\right)$

$E_{1}-\left(B_{1}+B_{2}+B_{3}\right)$

$E_{1}-\left(A_{1}+B_{1}+B_{2}\right)$

$E_{1}-\left(A_{1}+A_{2}+B_{1}\right)$

$E_{1}-\left(C_{1}+2 C_{2}\right)$ number of pairs $\{F,-F\}$

$\begin{array}{ll}E_{1}-\left(A_{4}+A_{5}+A_{6}\right) & 10 \\ E_{1}-\left(B_{4}+B_{5}+B_{6}\right) & 10 \\ & 90 \\ & 90 \\ & 132\end{array}$

There are 332 pairs $\{F,-F\}$ where $F$ is of type (3.13) and $\theta(F)=0 r$ times.

$$
332>275=\left(\begin{array}{c}
p+1 \\
3
\end{array}\right)+2\left(\begin{array}{c}
p+1 \\
2
\end{array}\right) \text {. }
$$


Case. $s=6, t \geqslant 9, p=t+4$.

Order of vanishing of F

$E_{1}-\left(A_{1}+A_{2}+A_{3}\right) \quad E_{1}-\left(A_{4}+A_{5}+A_{6}\right)$

10

$E_{1}-\left(B_{1}+B_{2}+B_{3}\right)$

$\left(\begin{array}{c}t \\ 3\end{array}\right)$

$E_{1}-\left(A_{1}+B_{1}+B_{2}\right)$

There are

$$
10+\left(\begin{array}{l}
t \\
3
\end{array}\right)+6\left(\begin{array}{l}
t \\
2
\end{array}\right)+15 t+2\left(\begin{array}{c}
t+6 \\
2
\end{array}\right)
$$

pairs $\{F,-F\}$ where $F$ is of type (3.13) and $\theta(F)=0 r$ times. This number is

$$
\left(\begin{array}{c}
p+2 \\
3
\end{array}\right)+2\left(\begin{array}{c}
p+2 \\
2
\end{array}\right)-10>\left(\begin{array}{c}
p+1 \\
3
\end{array}\right)+2\left(\begin{array}{c}
p+1 \\
2
\end{array}\right) \text {. }
$$

Case. $s \geqslant 9, t \geqslant 9, p=s+t-2$.

Order of vanishing of

the theta function



$$
\begin{aligned}
& E_{1} \\
& E_{1}-\left(C_{1}+C_{2}+C_{3}\right) \\
& E_{1}-\left(2 C_{1}+C_{2}\right)
\end{aligned}
$$

There are $\left({ }_{3}^{p+2}\right)+2\left({ }_{2}^{p+2}\right)$ pairs $\{F,-F\}$ where $F$ is of type (3.13) and $\theta(F)=0 r$ times. This number is greater than $\left({ }_{3}^{p+1}\right)+2\left({ }_{2}^{p+1}\right)$.

All the cases of the lemma have been exhausted. Q.E.D.

The second aspect of the vanishing properties to be considered is some group theoretic properties of the $(1 / 6)$-periods. The whole set of $(1 / 6)$-periods where we have information about the vanishings of the theta functions is the coset $E_{2}+Q_{\text {, }}$, which in this case equals $E_{1}+\mathcal{G}$ since $s \equiv t \equiv 0(\bmod 3)$. Let $\left\langle C_{1}-C_{2}\right\rangle$ be a cyclic subgroup of $\mathcal{G}$ of order three. Then the coset

$$
E_{1}-\left\langle C_{1}-C_{2}\right\rangle=\left\{E_{1}, E_{1}-\left(C_{1}+2 C_{2}\right), E_{1}-\left(2 C_{1}+C_{2}\right)\right\}
$$

is a set of zeros of the theta function of orders $r+1, r$, and $r$, respectively. Moreover, none of these $(1 / 6)$-periods is a $(1 / 2)$-period. For if one were, then $2 E_{1} \equiv 0, C_{1}+2 C_{2}$, or $2 C_{1}+C_{2}$. But $2 E_{1} \equiv \Sigma A_{j} \equiv \Sigma B_{j} \not \equiv 0$ by (3.7) and Lemma 3.1. We summarize these results in the following lemma. The results are also true for $r=1$.

Lemma 3.4. Let $p=3 r+1, r \geqslant 1$. Suppose $W_{p}$ is a cyclic trigonal Riemann surface with $s>0$. Then there is a cyclic subgroup of $\mathcal{G},\langle\eta\rangle$, so that the theta function vanishes on the points of the coset $E_{1}-\langle\eta\rangle$ to orders $r+1, r$, and $r$. Moreover, none of the (1/6)-periods of the coset are (1/2)-periods. 
Case 2. $p \equiv 2(\bmod 3)$. Let $p=3 r+2, r \geqslant 1$. In this case the theta function vanishes at $E_{1}-C_{k}, k=1,2, \ldots, p+2$, to order $r+1$. However, if $s=2$ we have $2 E_{1}=A_{1}+A_{2}$ or $\left(E_{1}-A_{1}\right)+\left(E_{1}-A_{2}\right)=0$. This is compensated for by the existence of a half-period $E_{2}$ where the theta function vanishes to order $r$.

LEMMA 3.5. Let $p=3 r+2, r \geqslant 1$. If $W_{p}$ is cyclic trigonal with $s>2$ then there are $p+2$ pairs $\{F,-F\}$ where $F$ is of the form $E_{1}-C_{1}$ and the theta function vanishes to order $r+1$ at $F$. If $s=2$ there are only $p+1$ such pairs, but there is also a half-period $E_{2}$, where $3 E_{2} \equiv 3 F$, and the theta function vanishes to order $r$ at $E_{2}$.

Lemma 3.6. Let $p=3 r+2, r \geqslant 1$. Suppose $W_{p}$ is a cyclic trigonal Riemann surface. Then there are (1/6)-periods $E_{1}-C_{k}, k=1,2, \ldots, p+2$, and cyclic subgroups of $\mathcal{G}$, $\langle\eta\rangle$, so that the theta function vanishes at the points of the coset $E_{1}-C_{k}+\langle\eta\rangle$ to orders $r+1, r+1$, and $r$. Moreover, $\langle\eta\rangle$ can be chosen so that none of the (1/6)-periods in this coset is a (1/2)-period.

Proof. Fix any $C_{k}$ and let $\eta=C_{k}-C_{l}$. The points of the coset are $E_{1}-C_{k}$, $E_{1}-C_{l}$, and $E_{1}-\left(2 C_{k}+2 C_{l}\right)$. The theta function vanishes as indicated. For any of these to be (1/2)-periods we must have $2 E_{1} \equiv C_{k}, C_{l}$, or $C_{k}+C_{l}$. This can only happen if $s=2$ and $\left\{C_{k}, C_{l}\right\}=\left\{A_{1}, A_{2}\right\}$ so if $s=2$ and $C_{k}$ is one of the $A$ 's, $C_{l}$ must be chosen different from the other $A$. Q.E.D.

Case 3. $p \equiv 3(\bmod 3)$. Let $p=3 r+3, r \geqslant 1$. (1/6)-periods of type (3.9) with the highest vanishings for theta are

$$
E_{1}-\left(C_{k}+C_{l}\right) \text { or } E_{1}-\left(2 C_{k}\right)
$$

There are

$$
\left(\begin{array}{c}
p+2 \\
2
\end{array}\right)+\left(\begin{array}{c}
p+2 \\
1
\end{array}\right)=\left(\begin{array}{c}
p+3 \\
2
\end{array}\right)
$$

such (1/6)-periods. Only if $s=1$ and $C_{k}=A_{1}$ is a (1/6)-period of type (3.14) a (1/2)-period, namely $E_{1}-2 A_{1}$. In all other cases the (1/6)-periods are not $(1 / 2)$ periods. The theta function vanishes to order $r+1$ at (1/6)-periods of type (3.14). As in previous cases we want to count the number of pairs $\{F,-F\}$ where $F$ is of type (3.14). Again we state the result in a form useful for the characterizations of $\S 4$.

Lemma 3.7. Let $p=3 r+3, r \geqslant 1$. Suppose $W_{p}$ is a cyclic trigonal Riemann surface. If $s>1$ then there are more than $\left({ }_{2}^{+2}\right)$ pairs $\{F,-F\}$, where $F$ is of type (3.14) and the theta function vanishes to order $r+1$ at $F$. If $s=1$ there are precisely $\left({ }_{2}^{p+2}\right)$ such pairs and, additionally, there is a (1/2)-period $E_{2}$ so that $3 E_{2} \equiv 3 F$ and $\theta\left(E_{2}\right)=0 r+1$ times.

Proof. Again we shall tabulate the results, being sure to account for all $\left({ }_{3}^{p+2}\right)$ (1/6)-periods of type (3.14). 
Case. $s=1, t \geqslant 7, p=t-1$.

Order of vanishing of the theta function

$$
\begin{array}{lll}
r+1 & E-2 A_{1} & E-2 A_{1} \\
r+1 & E-2 B_{j} & E-\left(A_{1}+B_{j}\right) \\
r+1 & E-\left(B_{i}+B_{j}\right) &
\end{array}
$$

F
$-F$ number of pairs

$\{F,-F\}$

There are

$$
t+\left(\begin{array}{l}
t \\
2
\end{array}\right)=\left(\begin{array}{c}
t+1 \\
2
\end{array}\right)
$$

pairs $\{F,-F\}$ of $(1 / 6)$-periods and also the (1/2)-period $E_{1}-2 A_{1}$. At all these points the theta function vanishes to order $r+1$.

Case. $s=4, t=4, p=6$.

Order of vanishing of

F $-F$

1

$t$

$-F \quad$ number of pairs

$\{F,-F\}$

2
2
2
2

$E-2 C_{k}$

$E_{1}-\left(A_{1}+A_{2}\right) \quad E_{1}-\left(A_{3}+A_{4}\right)$

$E_{1}-\left(B_{1}+B_{2}\right) \quad E_{1}-\left(B_{3}+B_{4}\right)$

$E_{1}-\left(A_{1}+B_{1}\right)$

Thus there are 30 such pairs and

$$
30>28=\left(\begin{array}{c}
p+2 \\
2
\end{array}\right), \quad p=6 .
$$

Case. $s=4, t>4, p=t+2$.

Order of vanishing of the theta function

$$
\begin{aligned}
& r+1 \\
& E_{1}-2 C_{k} \\
& r+1 \\
& E_{1}-\left(A_{1}+A_{2}\right) \\
& r+1 \\
& E_{1}-\left(B_{1}+B_{2}\right) \\
& r+1 \\
& E_{1}-\left(A_{1}+B_{1}\right)
\end{aligned}
$$

$$
\text { F }-F
$$

number of pairs

$\{F,-F\}$

Thus there are $\left({ }_{3}^{p+2}\right)-3$ such pairs and this number is greater than $\left(\begin{array}{c}p+2 \\ 2\end{array}\right)$.

Case. $s>4, t \geqslant s$. In this case there are $\left({ }_{2}^{p+3}\right)$ such pairs.

All cases have been exhausted. Q.E.D.

Now consider

$$
E_{1}-\left(C_{k}+C_{l}\right)+\left\langle C_{k}-C_{l}\right\rangle=\left\{E_{1}-2 C_{k}, E_{1}-\left(C_{k}+C_{l}\right), E_{1}-2 C_{l}\right\} .
$$

Only if $s=1$ and $C_{k}$ or $C_{l}=A_{1}$ are any of these (1/6)-periods a (1/2)-period. The theta function vanishes at each of these (1/6)-periods to order $r+1$. 
Lemma 3.8. Let $p=3 r+3, r \geqslant 1$. Suppose $W_{p}$ is a cyclic trigonal Riemann surface. Then there are (1/6)-periods $E_{1}-\left(C_{k}+C_{l}\right)$ and cyclic subgroups of $\mathcal{G},\langle\eta\rangle\left(\eta=C_{k}\right.$ $-C_{l}$ ) so that the theta function vanishes to order $r+1$ at the points of the coset $E_{1}-\left(C_{k}+C_{l}\right)+\langle\eta\rangle . C_{k}, C_{l}$ and $\langle\eta\rangle$ can be chosen so that none of the (1/6)-periods in the coset are (1/2)-periods.

Case 4. $p \equiv 1(\bmod 3)$ and $s=0$. Let $p=3 r+1, r \geqslant 2$. In this case, $E_{1}\left(=E_{2}\right)$ is a (1/2)-period and the theta function vanishes to order $r+1$ at $E_{1}$. At (1/6)-periods of type

$$
E_{1}-\left(B_{j}+B_{k}+B_{l}\right) \text { or } E_{1}-\left(2 B_{j}+B_{k}\right) \text { or } E_{1}-\left(2 B_{j}+2 B_{k}+2 B_{l}\right)
$$

the theta function vanishes to order $r$. Since

$$
\left(E_{1}-\left(B_{j}+B_{k}+B_{l}\right)\right)+\left(E_{1}-\left(2 B_{j}+2 B_{k}+2 B_{l}\right)\right) \equiv 0
$$

and

$$
\left(E_{1}-\left(2 B_{j}+B_{k}\right)\right)+\left(E_{1}-\left(B_{j}+2 B_{k}\right)\right) \equiv 0
$$

we see there are

$$
\left(\begin{array}{c}
p+2 \\
3
\end{array}\right)+\left(\begin{array}{c}
p+2 \\
2
\end{array}\right)=\left(\begin{array}{c}
p+3 \\
3
\end{array}\right)
$$

pairs $\{F,-F\}$ of $(1 / 6)$-periods where $F$ is of type (3.15) and the theta function vanishes at $F$ to order $r$.

LeMma 3.9. Let $p=3 r+1, r \geqslant 2$. Suppose $W_{p}$ is cyclic trigonal with $s=0$. Then there are $\left(c_{3}^{+3}\right)$ pairs $\{F,-F\}$ of $(1 / 6)$-periods so that $\theta(F)=0$ times. None of these (1/6)-periods are (1/2)-periods. Also there is a unique (1/2)-period $E_{1}$ so that $\theta\left(E_{1}\right)=0 r+1$ times and $3 E_{1} \equiv 3 F$.

Now consider subgroups of $\mathcal{G}$ of order nine as follows: $\left\langle B_{1}-B_{2}, B_{1}-B_{3}\right\rangle$. The coset $E_{1}-\left\langle B_{1}-B_{2}, B_{1}-B_{3}\right\rangle$ is $\left\{E_{1}, E_{1} \pm\left(B_{1}+B_{2}+B_{3}\right), E_{1} \pm\left(B_{1}+2 B_{2}\right), E_{1}\right.$ $\left.\pm\left(B_{2}+2 B_{3}\right), E_{1} \pm\left(B_{3}+2 B_{1}\right)\right\}$. The theta function vanishes at the nine points of this coset to orders $r+1, r, r, \ldots, r$. Such a coset will be called a 9-coset.

LEMMA 3.10. There are $\left({ }_{3}^{p+2}\right)$ 9-cosets.

It should be pointed out that our discussion of high order vanishings of the theta function does not include a proof that we have accounted for all the high order vanishings (to order $r$ or $r+1$ as the case may be). However, in Lemmas 3.3, 3.5, 3.7, and 3.9 we have accounted for enough high order vanishings to characterize the existence of a cyclic trigonal automorphism, as we shall see in $\$ 4$.

To see that we have, in fact, all the high order vanishings, one could make a complete table of all the vanishing properties, a task we have decided to postpone. Alternately, we can argue as follows.

If $\theta(F)=0 k+1$ times, then there is (by Lemma 2.4) a $g_{p-1}^{k}=k g_{3}^{1}+D$, where $D=\sum \varepsilon_{i} c_{i}, \varepsilon_{i}=0,1,2$, and $\sum \varepsilon_{i}=p-1-3 k$. If $p=3 r+1$, then

$$
g_{3 r}^{r-1}=(r-1) g_{3}^{1}+c_{i}+c_{j}+c_{k}
$$


and $F($ or $-F)=E_{1}-\left(C_{i}+C_{j}+C_{k}\right)$ by (3.6). If $p=3 r+2$, then

$$
g_{3 r+1}^{r}=r g_{3}^{1}+c_{i}
$$

and $F($ or $-F)=E_{1}-C_{i}$ by (3.6). If $p=3 r+3$, then

$$
g_{3 r+2}^{r}=r g_{3}^{1}+c_{i}+c_{j}
$$

and $F$ (or $-F)=E_{1}-\left(C_{i}+C_{j}\right)$ by (3.6). Thus, all the highest order vanishings are accounted for in the previous tables.

4. The characterizations for $p \geqslant 5$. In this section we show that a Riemann surface whose theta function has the vanishing properties derived in $\S 3$ and the nonvanishing properties of $\$ 2$ is in fact cyclic trigonal. We consider the four cases separately. For each case we first prove the existence of a $g_{3}^{1}$. Then we show the $g_{3}^{1}$ has $p+2$ 3 -branch points; that is, it is cyclic trigonal. The cases are numbered as in $\S 3$.

4.1. Let $p=3 r+1, r \geqslant 2$. We assume $W_{p}$ has the vanishing and nonvanishing properties of a cyclic trigonal Riemann surface with $s \neq 0$; that is, $W_{p}$ does not admit a half-canonical $g_{3 r}^{r}$.

LEMMA 4.1.1. $W_{p}$ admits $a g_{3}^{1}$.

Proof. Assume $W_{p}$ does not admit a $g_{3}^{1}$.

There are three (1/6)-periods in $J\left(W_{p}\right), E_{1}, E_{1}+\eta, E_{1}+2 \eta$, none of which is a (1/2)-period, where the theta function vanishes to order $r+1, r$, and $r$ (Lemma 3.4).

Corresponding to the theta function vanishing at $E_{1}$ there is a $g_{3 r}^{r}$ on $W_{p}$ which is not $r g_{3}^{1}$, by assumption. If $g_{3 r}^{r}$ were composite then there would be a two-sheeted cover $W_{p} \rightarrow W_{q}$ and a complete $g_{(3 r-\varepsilon) / 2}^{r}$ on $W_{q}$ which lifts to the nonfixed points of $g_{3 r}^{r}$. By Clifford's theorem, $g_{(3 r-\varepsilon) / 2}^{r}$ is not special and so by the Riemann-Roch theorem $q=(r-\varepsilon) / 2$. Thus $6 q=3 r-3 \varepsilon=p-1-3 \varepsilon$. But the nonvanishing properties of the theta function (Lemma 2.7) exclude this value of $q$. Consequently $g_{3,}^{r}$ must be simple.

On the smooth three-sheeted cover $W_{3 p-2}$ of $W_{p}$ corresponding to the subgroup $\langle\eta\rangle$ in $J\left(W_{p}\right)$ there is a $g_{9 r}^{3 r}$ obtained by lifting the three linear series corresponding to the theta function vanishing at $E_{1}, E_{1}+\eta, E_{1}+2 \eta$ (Lemma 2.6). $g_{9 r}^{3 r}$ must be simple.

We assert that $g_{9 r}^{3 r}$, which is $(1 / 6)-3 K$, is not $(1 / 2)-K$. On $W_{p}$ there is a $(1 / 6)-3 K$ $h_{3 r}^{r}$ so that $g_{3 r}^{r}+h_{3 r}^{r} \equiv K$. If $g_{9 r}^{3 r}$ is $(1 / 2)-K$ then $g_{3 r}^{r}$ and $h_{3 r}^{r}$ lift to equivalent divisors on $W_{3 p-2}$. Thus $h_{3 r}^{r}$ corresponds to one of the periods in $E_{1}+\langle\eta\rangle$, which must, in fact, be $E_{1}$, since the theta function vanishes to order $r+1$ there. This means $E_{1}$ is a (1/2)-period, a contradiction.

Thus $g_{9 r}^{3 r}$ is not $(1 / 2)-K$. By Lemmas 2.3 and $2.8, W_{3 p-2}$ admits a $g_{3}^{1}$. $W_{p}$ admits a $g_{3}^{1}$ after all. Q.E.D.

REMARK. One can find a similar proof without going to $W_{3 p-2}$, but eliminating the possibility of a $g_{4}^{1}$ on $W_{p}$ turns out to be extremely tedious.

The referee points out that in applying Lemmas 2.3 and 2.8, a remark excluding the possibility of more than one $g_{4}^{1}$ on $W_{3 p-2}$ is in order. Two $g_{4}^{1}$ 's on $W_{3 p-2}$ would imply $W_{3 p-2}$ is $(0-\mathrm{H})$ or $(1-\mathrm{H})$ since $3 p-2 \geqslant 19$. Therefore $W_{p}$ would also be $(0-\mathrm{H})$ or $(1-\mathrm{H})$. But this is excluded by the nonvanishing properties. 
Now we show $W_{p}$ is cyclic trigonal by showing that the covering $W_{p} \rightarrow \mathbf{P}^{\prime}$ given by $g_{3}^{1}$ has $p+2$ 3-branched points.

Consider a pair of $(1 / 6)-3 K$ linear series, $g_{3 r}^{r-1}, h_{3 r}^{r-1}$, whose sum is canonical, corresponding to the theta function vanishing at a pair $\{F,-F\}$ to order $r$. By Lemma 2.4 at least one of them is $(r-1) g_{3}^{1}+x_{1}+x_{2}+x_{3}$ for three points $x_{1}, x_{2}, x_{3}$ on $W_{p}$. Since one of the two, $g_{3 r}^{r}$ or $h_{3 r}^{r}$, is $r g_{3}^{1}$ and $3 g_{3 r}^{r} \equiv 3 h_{3 r}^{r} \equiv 3 g_{3 r}^{r-1}$, we see that

$$
3\left(x_{1}+x_{2}+x_{3}\right) \equiv 3 g_{3}^{1}=g_{9}^{3+\varepsilon} .
$$

If $\varepsilon \geqslant 1$ then $W_{p}$ admits a simple $g_{9}^{4}$ and, by Castelnuovos' theorem, (Lemma 2.2), it follows that $p=7$. But if $p=7, g_{9}^{4}+g_{3}^{1} \equiv K$ so $4 g_{3}^{1}=K$. $2 g_{3}^{1}$ is $(1 / 2)-K$, a possibility excluded by hypothesis $(s>0)$. Consequently, $3 g_{3}^{1} \equiv g_{9}^{3}$, which is complete and composite.

LEMMA 4.1.2. $3 x_{1} \equiv 3 x_{2} \equiv 3 x_{3} \equiv g_{3}^{1}$.

Proof. Case 1. Suppose $x_{1} \neq x_{2} \neq x_{3} \neq x_{1} .3 x_{1}+3 x_{2}+3 x_{3}$ is the sum of 3 divisors in $g_{3}^{1}$ and $x_{1}+x_{2}+x_{3}$ is not a divisor in $g_{3}^{1}$ since $g_{3 r}^{r-1}$ is complete. Suppose $x_{1}+x_{2}$ is in one divisor $D_{1}$ of $g_{3}^{1}$ and $x_{3}$ is in $D_{2}$. Then $D_{1}+D_{2} \subset 3\left(x_{1}+x_{2}+x_{3}\right)$, and it follows that $3 x_{3}=D_{2}$ and $3\left(x_{1}+x_{2}\right)=2 D_{1}$. By relabeling if necessary we see that $2 x_{1}+x_{2}=D_{1}$, so $x_{1}+2 x_{2}=D_{1}$ and $x_{1}=x_{2}$, a contradiction. Consequently, $x_{1}, x_{2}$ and $x_{3}$ must lie in three distinct divisors of $g_{3}^{1}$. The lemma now follows in this case.

Case 2. Suppose $x_{1}=x_{2} \neq x_{3}$. Then $6 x_{1}+3 x_{3}=3 g_{3}^{1}$. If $x_{1}$ and $x_{3}$ are in the same divisor of $g_{3}^{1}$, we see that $g_{3}^{1}=2 x_{1}+x_{3}$, a contradiction. Consequently, $x_{1}$ and $x_{3}$ are in different divisors of $g_{3}^{1}$. The lemma now follows in this case.

Case 3. Suppose $x_{1}=x_{2}=x_{3}$. This is seen to be impossible. Q.E.D.

THEOREM 4.1.3. $W_{p}$ is cyclic trigonal.

Proof. Every vanishing of the theta function at a pair of (1/6)-periods $\{F,-F\}$ to order $r$ gives rise to either $(r-1) g_{3}^{1}+x_{1}+x_{2}+x_{3}$ where $3 x_{1} \equiv 3 x_{2} \equiv 3 x_{3} \equiv g_{3}^{1}$, or $(r-1) g_{3}^{1}+2 x_{1}+x_{2}$ where $3 x_{1} \equiv 3 x_{2} \equiv g_{3}^{1}$. Each $x_{i}$ is a 3-branch point of $g_{3}^{\prime}$. Notice that the existence of $7(=1+6)$ such pairs $\{F,-F\}$ implies only the existence of 3 such $x_{i}$ 's. The existence of $16(=4+12)$ such pairs $\{F,-F\}$ implies the existence of 4 such $x_{i}$ 's. In general if we have $\left(\begin{array}{l}n \\ 3\end{array}\right)+2\left(\begin{array}{l}n \\ 2\end{array}\right)$ such pairs $\{F,-F\}$ we can assume the existence of only $n$ such point $x_{i}$. But if we have more than $\left(\begin{array}{l}n \\ 3\end{array}\right)+2\left(\begin{array}{c}n \\ 2\end{array}\right)$ such pairs, there must be more than $n$ such $x_{i}$ 's. By Lemma 3.3 there are more than $\left({ }_{3}^{p+1}\right)+2\left({ }_{2}^{p+1}\right)$ such pairs. So $g_{3}^{1}$ has $p+23$-branch points. Q.E.D.

4.2. $p=3 r+2, r \geqslant 1$. We assume $W_{p}$ has the vanishing and nonvanishing properties of a cyclic trigonal Riemann surface.

LEMMA 4.2.1. $W_{p}$ admits $a g_{3}^{1}$.

Proof. We assume $W_{p}$ does not admit a $g_{3}^{1}$.

There are three (1/6)-periods in $J\left(W_{p}\right), E_{1}=C_{k}+\langle\eta\rangle\left(\eta=C_{k}-C_{l}\right)$ where the theta function vanishes to order $r+1, r+1$ and $r$, respectively. We can choose this coset so that none of the periods is a (1/2)-period (Lemma 3.6). 
Corresponding to the theta function vanishing at $E_{1}-C_{k}$ there is a $g_{3 r+1}^{r}$ which is not $r g_{3}^{1}+x$. If $g_{3 r+1}^{r}$ is composite, then one sees there is a two-sheeted cover $W_{p} \rightarrow W_{q}$ and a $g_{(3 r+1-\varepsilon) / 2}^{r}$ on $W_{q}$ which is nonspecial if $r \geqslant 2$ (by Clifford's theorem). By the Riemann-Roch theorem, $q=(r+1-\varepsilon) / 2$. Thus $6 q=3 r+3-$ $3 \varepsilon=p+1-3 \varepsilon$. If $p$ is even, $r$ is even, so $\varepsilon$ is odd; that is, if $p$ is even, $6 q \leqslant p-2$. If $p$ is odd. $6 q \leqslant p+1$. But the nonvanishing properties of the theta function exclude both these possibilities (Lemma 2.7). Thus if $r \geqslant 2, g_{3 r+1}^{r}$ is simple. (We will handle the case $r=1$ shortly.)

Now on the smooth 3-sheeted cover $W_{3 p-2}$ of $W_{p}$ corresponding to the subgroup $\langle\eta\rangle$ in $J\left(W_{p}\right)$, there is a $g_{9 r+3}^{3 r+1}$ obtained by lifing the three linear series corresponding to the theta function vanishing at $E_{1}-C_{k}+\langle\eta\rangle \cdot g_{9 r+3}^{3 r+1}$ is simple if $r \geqslant 2$ since $g_{3 r+1}^{r}$ is simple (Lemma 2.6).

If $r=1$ we show that $g_{12}^{4}$ on $W_{13}$ is simple. If not, it cannot be $4 g_{3}^{1}$ so $W_{13}$ is a two-sheeted cover of $W_{q}$ admitting a $g_{(12-\varepsilon) / 2}^{4}$. Thus $q=0,1$, or 2 . The covering $W_{13} \rightarrow W_{q}$ is strongly branched.

This implies $W_{5}$ is a two-sheeted cover of $W_{q^{\prime}}$, over which $W_{q}$ is a 3 -sheeted cover. This implies $q^{\prime}=0$ or 1 , a contradiction of the nonvanishing properties of the theta function for $W_{5}$.

Now we assert that $g_{9 r+3}^{3 r+1}$ is not $(1 / 2)-K$. Since $g_{3 r+1}^{r}$ is $(1 / 6)-K$, there is another (1/6)-3K $h_{3 r+1}^{r}$ so that $g_{3 r+1}^{r}+h_{3 r+1}^{r}=K$. If $g_{9 r+3}^{3 r+1}$ were $(1 / 2)-K$ then $g_{3 r+1}^{r}$ and $h_{3 r+1}^{r}$ lift to equivalent divisors on $W_{3 p-2}$. This implies $2\left(E_{1}-C_{h}\right) \in\langle\eta\rangle$, so one of the periods $E_{1}-C_{k}+\langle\eta\rangle$ is a half-period, contradiction (Lemma 3.6). Thus $g_{9 r+3}^{3 r+1}$ is simple and not $(1 / 2)-K$. So $W_{3 p-2}$ admits a $g_{3}^{1}$ and so does $W_{p}$ after all (Lemmas 2.3 and 2.8 and the referee's remarks following Lemma 4.1.1). Q.E.D.

Each pair $\{F,-F\}(6 F \equiv 0, \theta(F)=0 r+1$ times $)$ gives rise to a pair $g_{3 r+1}^{r}, h_{3 r+1}^{r}$ whose sum is canonical and at least one of these is $r_{3}^{1}+x_{i}$ by Lemma 2.4. Since there are at least $p+1$ such pairs $\left\{F_{i},-F_{i}\right\}$ such that $3 F_{i} \equiv 3 F_{j}$. we see that

$$
3\left(r g_{3}^{1}+x_{i}\right) \equiv 3\left(r g_{3}^{1}+x_{j}\right) \text { or } 3 x_{i} \equiv 3 x_{j} \equiv g_{3}^{1}
$$

since $g_{3}^{1}$ is unique.

THEOREM 4.2.2. $W_{p}$ is cyclic trigonal.

ProOF. By consulting the table of vanishings ( $\$ 3)$ we see that if $s \geqslant 5$ there are $p+2$ pairs $\left\{F_{i}-F_{i}\right\}$. Each $x_{i}$ is a 3 -branch point for $g_{3}^{1}$ so the proof is complete when there are $p+2$ such pairs.

If there are only $p+1$ such pairs $(s=2)$ we must find the $(p+2)$ nd 3 -branch point for $g_{3}^{1}$. We do this by showing that there is a pair $\{F,-F\}$ where the complementary linear series corresponding to $F$ and $-F$ both give rise to a 3-branch point.

There is a (1/2)-period $E_{2}$ so that $\theta\left(E_{2}\right)=0 r$ times and $3 E_{2} \equiv 3 F_{i}$ for all pairs $\left\{F_{i},-F_{i}\right\}$. This half-period gives rise to a $(1 / 2)-K$ linear series $g_{3 r+1}^{r-1}$ which is $(r-1) g_{3}^{1}+D_{4}, D_{4}$ a divisor of degree four. Since $3 g_{3 r+1}^{r-1}=(3 r+1) g_{3}^{1}$, we see that $3 D_{4} \equiv 4 g_{3}^{1} \equiv g_{12}^{4+\varepsilon}$. If $\varepsilon \geqslant 1$ then $W_{p}$ admits a simple $g_{12}^{5}$, so $p \leqslant 10$ by Castelnuovos' inequality. 
Assume $p \geqslant 11(r \geqslant 3)$.

$$
D_{4}=t+x+y+z \text { and } 3 t+3 x+3 y+3 z \equiv 4 g_{3}^{1}=g_{12}^{4} \text {. }
$$

The latter series is complete and composite. We show that (by relabeling if necessary) $t=x$ and $y=z$ and $3 t \equiv 3 y \equiv g_{3}^{1}$. First observe that no divisor of $g_{3}^{1}$ can be made up of three of the points of $D_{4}$ since $g_{3 r+1}^{r-1}$ is complete.

Now suppose the four points of $D_{4}$ lie in four distinct divisors of $g_{3}^{1}$. Then one sees that $3 t \equiv 3 x \equiv 3 y \equiv 3 z$. But

$$
2 g_{3 r+1}^{r-1}=K \text { or } 2 t+2 x+2 y+2 z+(2 r-2) g_{3}^{1}=K .
$$

Since two of the points of $3 t$ are in $2 t+2 x+2 y+2 z$, a special divisor, and $(2 r-2) g_{3}^{1}$ is without fixed points, we see that $t$ is a point of $2 x+2 y+2 z$, a contradiction.

Next suppose $t+x$ lies in a divisor of $g_{3}^{1}$. Since $y+z$ cannot contain a point of this divisor we see that $2 t+2 x$ must contain a divisor of $g_{3}^{1}$. Suppose $2 t+x$ is such a divisor. Then $t+2 x+3 y+3 z \equiv 3 g_{3}^{1}$. It follows that $t+2 x$ is also a divisor in $g_{3}^{1}$, so $t=x, 3 t \equiv g_{3}^{1}$. Then $3 y+3 z \equiv 2 g_{3}^{1}$. By an entirely analogous argument we see that $y=z$ and $3 y \equiv g_{3}^{1}$. Thus

$$
2(r-1) g_{3}^{1}+4 t+4 y=2 r g_{3}^{1}+t+y \equiv K .
$$

$r g_{3}^{1}+t$ and $r g_{3}^{1}+y$ are both (1/6)-3K linear series corresponding to the same pair $\{F,-F\}$ where the theta function vanishes $r+1$ times. Thus we have found the $(p+2)$ nd 3-branch point for $g_{3}^{1}$. This completes the proof for $p \geqslant 11$ when there are only $p+1$ pairs $\{F,-F\}$.

Now assume $r=2, p=8$ and there are only nine pairs $\{F,-F\}$. If in the previous part of the proof of this section $4 g_{3}^{1}=g_{12}^{4}$ is complete and composite, we can complete the proof as above (in fact, this case does not occur). If, however, $4 g_{3}^{1}=g_{12}^{5}$ then $K=g_{12}^{5}+t+y=4 g_{3}^{1}+t+y$. We know $3 K \equiv 14 g_{3}^{1}$ so $3 t+3 y=$ $2 g_{3}^{1}=g_{6}^{2}$, complete and composite. It follows that $3 t \equiv 3 y \equiv g_{3}^{1}$. Also $t \neq y$ since there is no $(1 / 2)-K g_{7}^{2}$. Consequently $2 g_{3}^{1}+t$ and $2 g_{3}^{1}+y$ give rise to the tenth 3-branch point for $g_{3}^{1}$.

Finally, if $r=1$ then $2 g_{3}^{1}=g_{6}^{2}$, which is special. Consequently $K \equiv 2 g_{3}^{1}+t+y$ and the proof is completed as in the previous paragraph. Q.E.D.

4.3. $p=3 r+3, r \geqslant 1$. We assume $W_{p}$ has the vanishing and nonvanishing properties of a cyclic trigonal Riemann surface.

LEMMA 4.3.1. $W_{p}$ admits $a g_{3}^{1}$.

Proof. Assume $W_{p}$ does not admit a $g_{3}^{1}$.

We can find (1/6)-periods in $J\left(W_{p}\right), E_{1}-2 C_{k}+\langle\eta\rangle\left(\eta=C_{k}-C_{l}\right)$, none of which is a (1/2)-period, where the theta function vanishes to order $r+1, r+1$, and $r+1$ (Lemma 3.8). On a smooth, cyclic, 3-sheeted cover of $W_{p}, W_{3 p-2}$, corresponding to $\langle\eta\rangle$, the three linear series on $W_{p}$ corresponding to the vanishings lift to a 1/6-3K $g_{9 r+6}^{3 r+2}$. We show that $g_{9 r+6}^{3 r+2}$ is simple. If it were composite it cannot be $(3 r+2) g_{3}^{1}$ since $W_{p}$ does not admit a $g_{3}^{1}$. Consequently $W_{3 p-2}$ is a two-sheeted cover of $W_{q^{\prime}}$ which admits a simple $g_{(9 r+6-\varepsilon) / 2}^{3 r+2}$. As before $q^{\prime}=(3 r+2-\varepsilon) / 2$ and 
$W_{3 p-2} \rightarrow W_{q^{\prime}}$ is strongly branched. Consequently, the automorphism of period three on $W_{3 p-2}$ descends to $W_{q^{\prime}}, W_{q^{\prime}} \rightarrow W_{q}$ is a smooth cyclic 3-sheeted cover, and $W_{p} \rightarrow W_{q}$ is two-sheeted. But

$$
6 q-6=2 q^{\prime}-2=3 r-\varepsilon=p-3-\varepsilon,
$$

or $6 q=p+3-\varepsilon$. If $p$ is even, $r$ is odd so $\varepsilon$ must be at least 3 ; that is, $6 q \leqslant p$. If $p$ is odd we have $6 q \leqslant p+3$. But the nonvanishing properties of $W_{p}$ (Lemma 2.7) exclude these two possibilities (just barely). Consequently $g_{9 r+6}^{3 r+2}$ is simple on $W_{3 p-2}$.

Now $g_{9 r+6}^{3 r+2}$ is not half-canonical by an argument analogous to that in previous sections.

Since $g_{9 r+6}^{3 r+2}$ is not half-canonical it follows as before that $W_{3 p-2}$ admits a $g_{3}^{1}$. Consequently $W_{p}$ does also. Q.E.D.

For each pair of (1/6)-periods (not $(1 / 2)-K)\left\{F_{i},-F_{i}\right\}$ where the theta function vanishes to order $r+1$, there is a pair of (1/6)-3K linear series whose sum is canonical, $g_{3 r+2}^{r}+h_{3 r+2}^{r} \equiv K$. We want to show that for at least one pair, $3 g_{3 r+2}^{r} \equiv$ $(3 r+2) g_{3}^{1} \equiv 3 h_{3 r+2}^{r}$. But the proof of Lemma 4.3.1 shows that on a suitable 3-sheeted cover of $W_{p} . W_{3 p-2}$, the lift of $g_{3 r+2}^{r}$ or $h_{3 r+2}^{r}$ must be $(3 r+2) g_{3}^{1}=g_{9 r+6}^{3 r+2}$ by Lemma 2.4. Dropping this equation to $W_{p}$ gives $(3 r+2) g_{3}^{1}=3 g_{3 r+2}^{r}$. Having proven this for one pair $\left\{F_{i},-F_{i}\right\}$, it holds for all linear series corresponding to all pairs since $3 F_{i} \equiv 3 F_{j}$.

THEOREM 4.3.2. $W_{p}$ is cyclic trigonal.

Proof. Every vanishing of the theta function at a pair $\left\{F_{i},-F_{i}\right\}, 6 F_{i} \equiv 0$, to order $r+1$ gives rise to either $r g_{3}^{1}+x+y$ or $r g_{3}^{1}+2 x$ where $3 x \equiv 3 y \equiv g_{3}^{1}$ since $3(x+y) \equiv 2 g_{3}^{1}$ or $6 x \equiv 2 g_{3}^{1}$. Each such $x$ or $y$ is a 3-branch point for $g_{3}^{1}$. If there are three such pairs $\left\{F_{i},-F_{i}\right\}$ there are at least 2 such 3-branch points. If there are $\left(\begin{array}{c}n+1 \\ 2\end{array}\right)$ such pairs, then there are at least $n$ such 3-branch points.

Now if $s \geqslant 4$ we know there are more than $\left({ }_{2}^{+2}\right)$ such pairs; consequently the $g_{3}^{1}$ has more than $p+13$-branch points.

If $s=1$ there are precisely $\left({ }_{2}^{p+2}\right)$ such pairs, so we look for the $(p+2)$ nd 3-branch point elsewhere. There is in this case a half period $E_{2}$ at which the theta function vanishes $r+1$ times and $3 E_{2} \equiv 3 F_{i}$. The corresponding $(1 / 2)-K$ linear series must be $\operatorname{rg}_{3}^{1}+x+y$, where $3 x \equiv 3 y \equiv g_{3}^{1}$ and $2 x+2 y$ is special. It follows that $x=y$ and $3 x \equiv g_{3}^{1}$. This is the additional 3-branch point for $g_{3}^{1}$. Q.E.D.

4.4. Let $p=3 r+1, r \geqslant 2$. We assume $W_{p}$ has the vanishing and nonvanishing properties of a cyclic trigonal Riemann surface with $s=0 . W_{p}$ admits a unique $(1 / 2)-K g_{3 r}^{r}$.

LEMMA 4.4.1. $W_{p}$ admits $a g_{3}^{1}$.

Proof. There is a 9-group $\langle\eta, \nu\rangle\left(\eta=C_{k}-C_{l}, \nu=C_{k}-C_{m}\right)$ so that

$$
\theta\left(E_{1}+\varepsilon_{1} \eta+\varepsilon_{2} \nu\right)=0
$$

$r$ times $\left(\varepsilon_{1}, \varepsilon_{2}=0,1,2\right.$, not both zero) while $\theta\left(E_{1}\right)=0 r+1$ times. On a smooth 9-sheeted cover of $W_{p}, W_{9 p-8}$, corresponding to $\langle\eta, \nu\rangle$ there is a $(1 / 2)-K g_{27 r}^{9 r}$. Since $r \geqslant 2$ and $9 p-8 \geqslant 55$, by Lemma $2.9 W_{9 p-8}$ must admit a $g_{3}^{1}$, a $g_{4}^{1}$ or a $\gamma_{3}^{1}$. The $g_{4}^{1}$ is 
excluded by Lemma 2.8, so $W_{9 p-8}$ has a 3-sheeted map to $W_{q^{\prime}}$ where $q=0$ or 1 . The map is strongly branched in either case so the group $Z_{3} \times Z_{3}$ descends to act on $W_{q^{\prime}}$. Since $W_{9 p-8} \rightarrow W_{q^{\prime}}$ is not an intermediate covering of $W_{9 p-8} \rightarrow W_{p}$, the entire group acts nontrivially on $W_{q^{\prime}}$. Then $q=1$ since the group cannot act on $\mathbf{P}^{1}$; that is, $W_{9 p-8}$ admits a $\gamma_{3}^{1}$.

Now suppose $W_{p}$ does not admit a $g_{3}^{1}$.

The quotient of $W_{1}^{\prime}(q=1)$ by $Z_{3} \times Z_{3}$ has genus one. Thus we obtain a configuration of coverings of Riemann surfaces as in Diagram $I$.

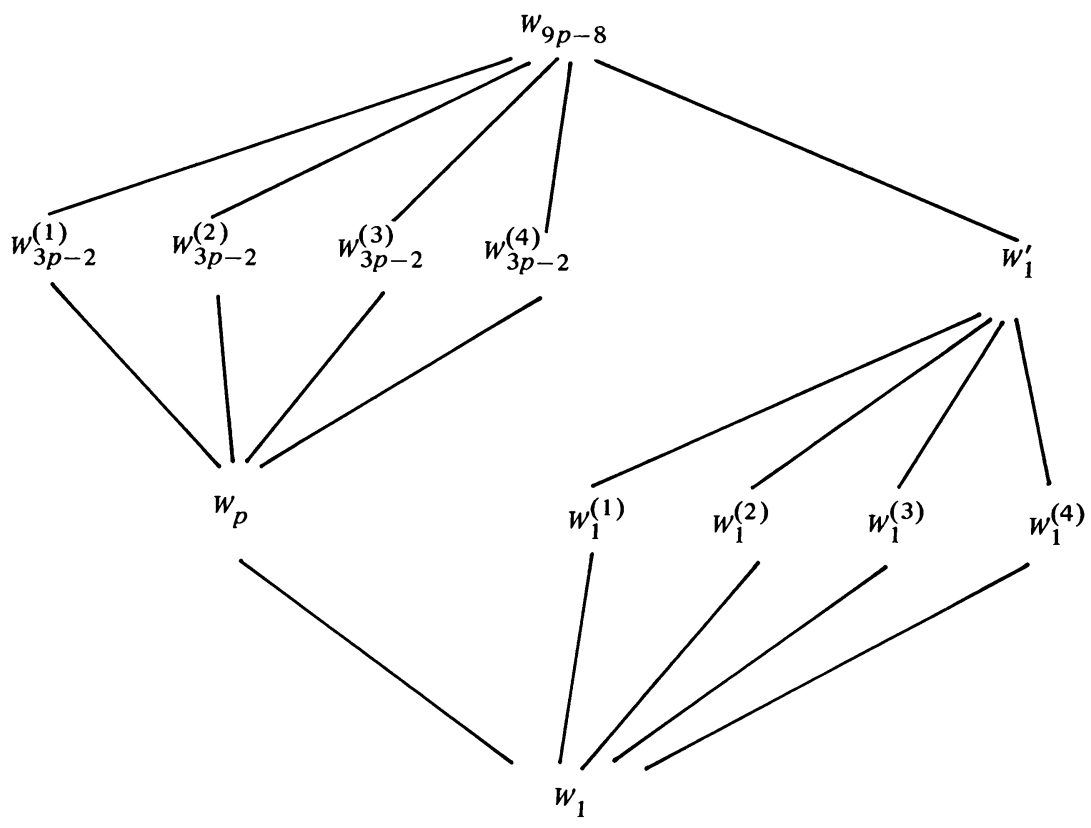

\section{DIAGRAM I}

We assert that $g_{3 r}^{r}$ on $W_{p}$ is lifted from a $g_{r}^{r-1}$ on $W_{1}$. Once we know this, then by Lemma 2.10 we know $W_{p}$ admits a complete $(1 / 2)-K h_{3 r}^{r-1}$. This contradicts the nonvanishing properties of $W_{p}$ (Lemma 2.11) and the proof will be concluded.

To prove the assertion we remind ourselves that $g_{27 r}^{9 r}=\left|g_{27 r}^{9 r-1}\right|$ on $W_{9 p-8}$, where $g_{27 r}^{9 r-1}$ is the lift from $W_{1}^{\prime}$ of a $g_{9 r}^{9 r-1}$. The lift of $g_{3 r}^{r}$ from $W_{p}$ to $W_{9 p-8}$ is a $g_{27 r}^{r}$, also included in $g_{27 r}^{9 r}$. By counting dimensions, we see there is a divisor $D$ common to $g_{27 r}^{9 r-1}$ and $g_{27 r}^{r}$. D is lifted to $W_{9 p-8}$ from $W_{p}$ and from $W_{1}^{\prime}$; consequently, it is the lift from $W_{1}$ of a divisor $D_{0}$ of degree $r$. On $W_{1},\left|D_{0}\right|=g_{r}^{r-1}$ and $D_{0}$ lifted to $W_{p}$ is in $g_{3 r}^{r}$. This proves the assertion. Q.E.D.

(We thank the referee for greatly clarifying the proof of this lemma.)

Notice that the above proof only concludes that the genus of $W_{1}$ is zero. $W_{1}^{\prime}$ must still be a torus since it admits a $Z_{3} \times Z_{3}$ of automorphisms. Consequently, everything in Diagram I is correct except the genera of $W_{1}$ and three of the $W_{1}^{(i)}$. Since we will need this fact shortly, we include Diagram II which exhibits the correct genera. 


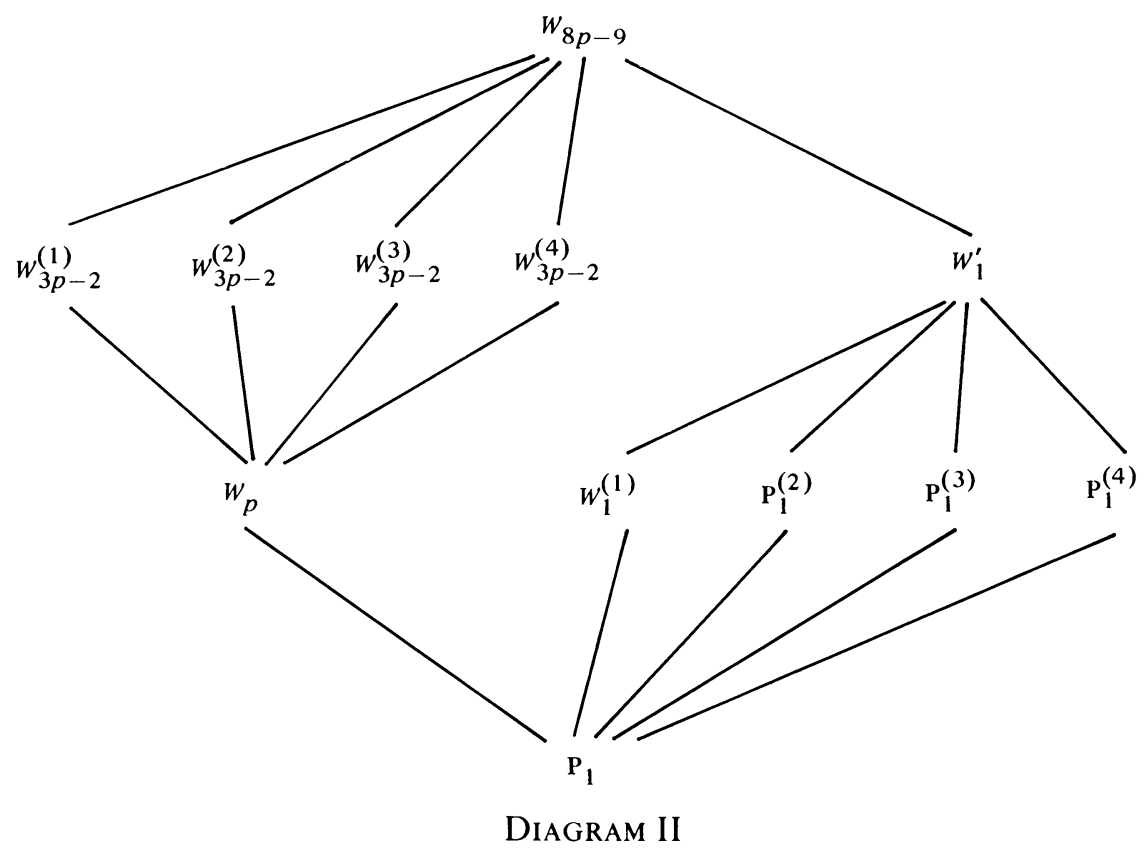

Every pair of $(1 / 6)$-periods $\{F,-F\}$ where the theta function vanishes to order $r$ leads to a $(1 / 6)-3 K$ linear series $(r-1) g_{3}^{1}+x_{1}+x_{2}+x_{3}$. Since $3 F \equiv 3 E_{1}$ and $g_{3 r}^{r}=r g_{3}^{1}$, we see that $3\left(x_{1}+x_{2}+x_{3}\right) \equiv 3 g_{3}^{1}=g_{9}^{3}$, complete and composite, unless $p=7$. It follows that $3 x_{1} \equiv 3 x_{2} \equiv 3 x_{3}=g_{3}^{1}$ if $p>7$, and from this, by elementary counting, we can derive the $p+23$-branch points for $g_{3}^{1}$ as before. For $p=7$ this does not seem to work, so we use an argument that will work for all $r \geqslant 2$.

For each 9-coset we obtain a surface $W_{9 p-8}$ and a configuration of covering as in Diagram II. We show that each 9-coset corresponds to a unique triple of 3-branch points in $g_{3}^{1}$. Since there are $\left({ }_{3}^{p+2}\right) 9$-cosets, elementary counting now insures the existence of $p+23$-branch points for $g_{3}^{1}$.

Consider the subdiagram

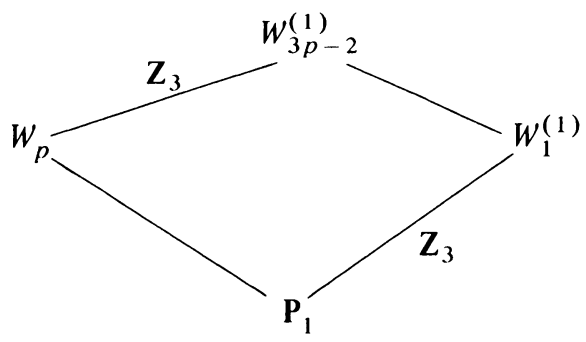

DiAgRAM III

The $Z_{3}$ 's indicate cyclic covers. $W_{1}^{(1)} \rightarrow \mathbf{P}_{1}$ is branched over 3 points in $\mathbf{P}_{1}$, say $\delta_{1}$, $\delta_{2}$ and $\delta_{3}$. But the cover $W_{3 p-2}^{(1)} \rightarrow W_{p}$ is smooth. This implies $W_{p} \rightarrow \mathbf{P}_{1}$ has three 3-branch points over $\delta_{1}, \delta_{2}$, and $\delta_{3}$. $W_{3 p-2}^{(1)}$ is uniquely determined by the cover $W_{p} \rightarrow \mathbf{P}_{1}$ and the points $\delta_{1}, \delta_{2}$, and $\delta_{3}$ since it is the minimal covering of the covers 
$W_{p} \rightarrow \mathbf{P}_{1}$ and $W_{1}^{(1)} \rightarrow \mathbf{P}_{1}$. Also, the whole of the configuration of coverings in Diagram II is determined by these two coverings since the 3 -sheeted coverings $\mathbf{P}_{1}^{(i)} \rightarrow \mathbf{P}_{1}$ are determined by pairs of the three points $\delta_{1}, \delta_{2}$, and $\delta_{3}$. This completes the proof.

THEOREM 4.4.2. $W_{p}$ is cyclic trigonal.

Remark. The proof shows that $W_{9 p-8} \rightarrow W_{1}^{\prime}$ is cyclic so $W_{9 p-8}$ admits an elementary abelian group of automorphisms of order 27.

5. One case for genus four. In this section we assume $W_{4}$ admits two distinct linear series, $g_{3}^{1}$ and $h_{3}^{1}$, whose sum in canonical. We characterize the cases when one or both of the trigonal coverings is cyclic.

First we assume $W_{4}$ is the Riemann surface for the equation

$$
y^{3}=\prod_{i=1}^{3}\left(x-\alpha_{i}\right) \prod_{j=1}^{3}\left(x-\beta_{j}\right)^{2},
$$

and we tabulate the vanishing properties of the theta function.

\begin{tabular}{cllc}
$\begin{array}{c}\text { Order of vanishing of } \\
\text { the theta function }\end{array}$ & \multicolumn{1}{c}{$F$} & \multicolumn{1}{c}{$-F$} & $\begin{array}{c}\text { number of pairs } \\
\{F,-F\}\end{array}$ \\
2 & $E_{1}$ & $E_{1}-\left(A_{1}+A_{2}+A_{3}\right)$ & 1 \\
1 & $E_{1}-\left(A_{1}+2 A_{2}\right)$ & $E_{1}-\left(2 A_{2}+A_{3}\right)$ & 3 \\
1 & $E_{1}-\left(B_{1}+2 B_{2}\right)$ & $E_{1}-\left(2 B_{2}+B_{3}\right)$ & 3 \\
1 & $E_{1}-\left(A_{1}+A_{2}+B_{1}\right)$ & $E_{1}-\left(A_{3}+2 B_{1}\right)$ & 9 \\
1 & $E_{1}-\left(A_{1}+B_{1}+B_{2}\right)$ & $E_{1}-\left(2 A_{1}+B_{3}\right)$ & 9
\end{tabular}

Thus there is one pair $\{F,-F\}$ when $\theta(F)=0$ twice and there are twenty-four pairs where $\theta(F)=0$ once.

Let us assume $h_{3}^{1}$ is the cyclic cover and

$$
g_{3}^{1}=\left|a_{1}+a_{2}+a_{3}\right|=\left|b_{1}+b_{2}+b_{3}\right| .
$$

Thus $u\left(h_{3}^{1}\right)+\kappa \equiv-E_{1}$ and

$$
u\left(g_{3}^{1}\right)+\kappa \equiv-\left(E_{1}-\left(A_{1}+A_{2}+A_{3}\right)\right) \equiv-\left(E_{1}-\left(B_{1}+B_{2}+B_{3}\right)\right) .
$$

The linear series of dimension zero are combinations of three of the $a$ 's and $b$ 's; e.g., if $g_{3}^{0}=\left|a_{1}+2 b_{3}\right|$ then

$$
u\left(g_{3}^{0}\right)+\kappa \equiv-\left(E_{1}-\left(A_{1}+2 B_{3}\right)\right) .
$$

The following observations will be useful in the characterizations to follow, so we gather them together in a lemma.

LEMMA 5.1. There are three kinds of $g_{3}^{0}$ 's corresponding to the (1/6)-periods in the preceding table.

(i) $g_{3}^{0}$ 's so that
(a) there is an $h_{3}^{0}$ and $g_{3}^{0}+h_{3}^{0} \equiv 2 g_{3}^{1}$;
(b) there is a $k_{3}^{0}$ and $g_{3}^{0}+k_{3}^{0} \equiv 2 h_{3}^{1}$. 
(ii) $g_{3}^{0}$ 's so that

(a) there is an $h_{3}^{0}$ and $g_{3}^{0}+h_{3}^{0} \equiv 2 g_{3}^{1}$;

(b) there does not exist a $k_{3}^{0}$ so that $g_{3}^{0}+k_{3}^{0} \equiv 2 h_{3}^{1}$

(iii) $g_{3}^{0}$ 's so that

(a) there does not exist an $h_{3}^{0}$ so that $g_{3}^{0}+h_{3}^{0} \equiv 2 g_{3}^{1}$;

(b) there is a $k_{3}^{0}$ so that $g_{3}^{0}+k_{3}^{0} \equiv 2 h_{3}^{1}$.

Proof. (i) Let $g_{3}^{0}=\left|a_{1}+2 a_{2}\right|, h_{3}^{0}=\left|a_{1}+2 a_{3}\right|$ and $k_{3}^{0}=\left|2 a_{1}+a_{2}\right|$.

(ii) Let $g_{3}^{0}=\left|a_{1}+a_{2}+b_{1}\right|$ and $h_{3}^{0}=\left|a_{3}+b_{2}+b_{3}\right|$.

(iii) Let $g_{3}^{0}=\left|a_{1}+2 b_{1}\right|$ and $k_{3}^{0}=\left|2 a_{1}+b_{1}\right|$. Q.E.D.

REMARK. The nonexistence of Lemma 5.1 (ii)(b) and (iii)(a) is reflected in nonvanishings. For instance, in (ii)(b) above, the (1/6)-period corresponding to the missing $k_{3}^{0}$ is one where the theta function does not vanish.

If $W_{4}$ admits two cyclic trigonal coverings there will be two sets of vanishings at (1/6)-periods as in the table. The only (1/6)-periods common to the two tables will be the $(1 / 6)$-periods where the theta function vanishes twice.

Now assume the theta function for $W_{4}$ has vanishings at $(1 / 6)$-periods as in the table. We will show that $W_{4}$ is a cyclic trigonal Riemann surface.

$W_{4}$ admits (1/6)-3K linear series $g_{3}^{1}, h_{3}^{1}$ and a collection of $g_{3}^{0}$ 's, and the triples of all of them are linearly equivalent. $2 g_{3}^{1}$ and $2 h_{3}^{1}$ are both $g_{6}^{2}$ 's which are complete and composite. The various $g_{3}^{0}$ 's will be denoted, as need be, by $h_{3}^{0}, k_{3}^{0}, 1_{3}^{0}, \ldots$

LEMma 5.2. Suppose $g_{3}^{0}+h_{3}^{0} \equiv 2 g_{3}^{1}$. Then one of the following cases must hold.

(i) $g_{3}^{0}=2 x+y, h_{3}^{0}=x+2 y, x \neq y$, and $3 x \equiv 3 y \equiv g_{3}^{1}$.

(ii) $g_{3}^{0}=2 x+y, h_{3}^{0}=y+2 z, x \neq y \neq z \neq x, g_{3}^{1}=|x+y+z|$ and $h_{3}^{1} \equiv 3 x \equiv$ $3 y \equiv 3 z$.

(iii) $g_{3}^{0}=x_{1}+x_{2}+x_{3} ; h_{3}^{0}=y_{1}+y_{2}+y_{3}$, the $x$ 's and $y$ 's are six distinct points on $W_{4}, g_{3}^{1} \equiv x_{1}+y_{2}+y_{3} \equiv y_{1}+x_{2}+x_{3}$ (by relabelling if necessary) and $h_{3}^{1} \equiv 3 x_{1}$ $\equiv 3 x_{2} \equiv 3 x_{3} \equiv 3 y_{1} \equiv 3 y_{2} \equiv 3 y_{3}$ (and so $W_{4}$ is cyclic trigonal).

Proof. First we show $g_{3}^{0}=3 x$ is impossible.

If $g_{3}^{0}=3 x$ and $h_{3}^{0}=y+z+t$, then $2 g_{3}^{1} \equiv 3 x+y+z+t$. By relabelling if necessary we see that $g_{3}^{1} \equiv x+y+z \equiv 2 x+t$. $t \neq x$ since $g_{3}^{1} \neq g_{3}^{0}$. But $3 g_{3}^{1} \equiv 3 g_{3}^{0}$ so $9 x \equiv 6 x+3 t$ or $3 x \equiv 3 t$. Thus $g_{3}^{0}$ has dimension greater than zero, a contradiction.

Now assume $g_{3}^{0}=2 x+y, x \neq y, h_{3}^{0}=z+s+t$. Thus $2 g_{3}^{1} \equiv 2 x+y+z+s+$ $t$. There are two cases.

(i) $g_{3}^{\prime} \equiv 2 x+z$ (by relabelling if necessary) $\equiv y+s+t$. Then

$$
3 g_{3}^{1} \equiv 6 x+3 z \equiv 3 g_{3}^{0} \equiv 6 x+3 y \quad \text { and } \quad 3 z \equiv 3 y \text {. }
$$

Since $y \neq z$ we see that $3 z \equiv 3 y \equiv k_{3}^{1}$. Suppose $k_{3}^{1}=h_{3}^{1}$. Then

$$
3 g_{3}^{1} \equiv 6 x+3 z \equiv 3 h_{3}^{1} \equiv 9 z, \quad 2 h_{3}^{1} \equiv 6 z \equiv 6 x,
$$

so $h_{3}^{1} \equiv 3 x$.

But $g_{3}^{1}=2 x+z$ so $h_{3}^{1}$ and $g_{3}^{1}$ have $2 x$ in common. This is impossible $(p=4)$. Consequently $3 z \equiv 3 y \equiv g_{3}^{1}$. It follows that $x=z$ and $y=s=t$. This is case (i) in the statement of the lemma. 
(ii) $g_{3}^{\prime} \equiv x+z+s \equiv x+y+t$ (by relabelling if necessary). We can assume without loss of generality that $z=y$ and $s=t$. Then

$$
3 g_{3}^{0} \equiv 6 x+3 y \equiv 3 g_{3}^{1} \equiv 3 x+3 y+3 t \quad \text { and } \quad 3 x \equiv 3 t \equiv k_{3}^{1} .
$$

If $k_{3}^{1}=g_{3}^{1}$ then $x=z=s$, a contradiction. Consequently $h_{3}^{1} \equiv 3 x \equiv 3 t .3 h_{3}^{1} \equiv 9 x \equiv$ $3 g_{3}^{0} \equiv 6 x+3 y$ so

$$
h_{3}^{1} \equiv 3 x \equiv 3 y \equiv 3 t \quad \text { and } \quad g_{3}^{1} \equiv x+y+t .
$$

This is case (ii) of the statement of the lemma.

(iii) Finally suppose $g_{3}^{0}=x_{1}+x_{2}+x_{3}$, three distinct points. Then $h_{3}^{0}=y_{1}+y_{2}$ $+y_{3}$, again three distinct points. (Otherwise, by cases (i) and (ii) $g_{3}^{0}$ could not have three distinct points.) Now $2 g_{3}^{1}=x_{1}+x_{2}+x_{3}+y_{1}+y_{2}+y_{3}$. By relabeling if necessary we can assume $g_{3}^{1} \equiv x_{1}+y_{2}+y_{3} \equiv y_{1}+x_{2}+x_{3}$. Since $3 g_{3}^{1} \equiv 3 h_{3}^{0}$, we see that $3 x_{1} \equiv 3 y_{1}$. Since $x_{1} \neq y_{1}$ it follows that $3 x_{1} \equiv h_{3}^{1}$. Consequently $2 h_{3}^{1} \equiv 3 x_{2}$ $+3 x_{3} \equiv 3 y_{2}+3 y_{3}$ and it follows that $h_{3}^{1} \equiv 3 x_{2} \equiv 3 x_{3} \equiv 3 y_{2} \equiv 3 y_{3}$. Now by examining all cases it follows by arguments already used that the six points $x_{1}$, $x_{2}, \ldots, y_{3}$ are all distinct. Q.E.D.

Lemma 5.3. Suppose $g_{3}^{0}+h_{3}^{0} \equiv 2 g_{3}^{1}, g_{3}^{0}+k_{3}^{0} \equiv K$, and $h_{3}^{0}+l_{3}^{0} \equiv K$; therefore $k_{3}^{0}+l_{3}^{0} \equiv 2 h_{3}^{1}$. Then

(a) If $g_{3}^{0}$ and $h_{3}^{0}$ satisfy case (i) of Lemma 5.2, then $k_{3}^{0}$ and $l_{3}^{0}$ satisfy cases (ii) or (iii) of Lemma 5.2 (with $g_{3}^{1}$ and $h_{3}^{1}$ interchanged).

(b) If $g_{3}^{0}$ and $h_{3}^{0}$ satisfy case (ii) of Lemma 5.2 then $k_{3}^{0}$ and $l_{3}^{0}$ satisfy case (i) of Lemma 5.2 (with $g_{3}^{1}$ and $h_{3}^{1}$ interchanged).

(c) If $g_{3}^{0}$ and $h_{3}^{0}$ satisfy case (iii) of Lemma 5.2 then $k_{3}^{0}$ and $l_{3}^{0}$ satisfy case (i) (with $g_{3}^{1}$ and $h_{3}^{1}$ interchanged ).

Proof. (a) We suppose $g_{3}^{0}=2 x+y, h_{3}^{0}=x+2 y, x \neq y$ and $3 x \equiv 3 y \equiv g_{3}^{1}$. The following is impossible.

$$
k_{3}^{0}=2 x^{\prime}+y^{\prime}, \quad l_{3}^{0}=x^{\prime}+2 y^{\prime}, \quad x^{\prime} \neq y^{\prime}, \quad 3 x^{\prime} \equiv 3 y^{\prime} \equiv h_{3}^{1} .
$$

For otherwise there is a canonical divisor $g_{3}^{0}+k_{3}^{0}=2 x+y+2 x^{\prime}+y^{\prime}$. Since $3 x=g_{3}^{1}$ it follows that $x=x^{\prime}$ or $x=y^{\prime}$ or $3 x \equiv 3 x^{\prime}$, a contradiction.

(b) Suppose $g_{3}^{0}=2 x+y, h_{3}^{0}=y+2 z, x \neq y \neq z \neq x, g_{3}^{1} \equiv x+y+z$, and $h_{3}^{1}$ $\equiv 3 x \equiv 3 y \equiv 3 z$. Then $k_{3}^{0}=2 x+z$ and $l_{3}^{0}=x+2 z$, which is case (i) of Lemma 5.2.

(c) Suppose $g_{3}^{0}=x_{1}+x_{2}+x_{3}, h_{3}^{0}=y_{1}+y_{2}+y_{3}$ where $g_{3}^{1} \equiv x_{1}+y_{2}+y_{3} \equiv y_{1}$ $+x_{2}+x_{3}$, and $h_{3}^{1} \equiv 3 x_{1} \equiv 3 x_{2} \equiv \cdots \equiv 3 y_{3}$. Then $k_{3}^{0}=y_{1}+2 x_{1}$, and $l_{3}^{0}=x_{1}+$ $2 y_{1}$, which is case (i) of Lemma 5.2. Q.E.D.

THEOREM 5.4. $W_{4}$ is cyclic trigonal.

Proof. We suppose the vanishing properties of the table but we suppose $W_{4}$ is not cyclic trigonal. Then case (iii) of Lemma 5.2 never occurs. Consequently the pairs $g_{3}^{0}$, $h_{3}^{0}$ and $k_{3}^{0}, l_{3}^{0}$ in Lemma 5.3 always satisfy cases (i) and (ii) of Lemma 5.2. Thus we can suppose

$$
g_{3}^{0}=2 x+y, \quad h_{3}^{0}=x+2 y, \quad k_{3}^{0}=2 x+z, \quad l_{3}^{0}=2 y+z,
$$

where $3 x \equiv 3 y \equiv 3 z \equiv h_{3}^{1}$ and $x+y+z \equiv g_{3}^{1}$. 
But each of the four linear series satisfies case (i) of Lemma 5.1. For instance $g_{3}^{0}+h_{3}^{0} \equiv 2 h_{3}^{1}$ and $g_{3}^{0}+(2 z+y) \equiv 2 g_{3}^{1}$. Thus cases (ii) and (iii) of Lemma 5.1 cannot occur. This contradiction proves the theorem. Q.E.D.

If $W_{4}$ admits two sets of vanishings as in the table, both $g_{3}^{1}$ and $h_{3}^{1}$ are cyclic. They will generate a noncyclic group of order nine. The four subgroups of order three have quotients of genus $0,0,2$, and 2 , that is, the other subgroups of order three are fixed point free.

The case of a cyclic trigonal $W_{4}$ admitting one half-canonical $g_{3}^{1}$ remains open. The above methods do not seem to work since $2 g_{3}^{1}=g_{6}^{3}$ is not composite. These methods appear to have even less applicability for cyclic trigonal $W_{3}$ 's.

6. Trigonal Riemann surfaces of low genus. Let $W_{2 p-1} \rightarrow W_{p}$ be a smooth two-sheeted covering. $J\left(W_{2 p-1}\right)$ is isogeneous to $J\left(W_{p}\right) \oplus A_{p-1}$, where $A_{p-1}$ is a principally polarized abelian variety, known as the Prym variety, for the covering [12]. If $W_{p}$ is a generic trigonal Riemann surface then $A_{p-1}$ is known to be a Jacobian (of a 4-sheeted cover of $\mathbf{P}_{1}$ ) [14]. In general $A_{p-1}$ is not a Jacobian. Thus if $W_{p}$ is trigonal, applying the Schottky-Jung-Farkas-Rauch theorem to the special theta relations for $A_{p-1}$ yields theta relations for $W_{p}$ which we shall call very special theta relations. We now describe them in a little more detail.

For appropriate choices of canonical homology bases on $W_{2 p-1}$ and $W_{p}$, the theorem of Schottky-Jung-Farkas-Rauch says

$$
\theta_{p}\left[\begin{array}{cc}
0 & \varepsilon \\
0 & \varepsilon^{\prime}
\end{array}\right] \theta_{p}\left[\begin{array}{cc}
0 & \varepsilon \\
1 & \varepsilon^{\prime}
\end{array}\right] / \theta_{p-1}^{2}\left[\begin{array}{c}
\varepsilon \\
\varepsilon^{\prime}
\end{array}\right]
$$

is a nonzero constant independent of the $(p-1)$-dimensional theta characteristic $\left[\begin{array}{l}{ }^{k} \\ \varepsilon^{\prime}\end{array}\right]$ [13]. $\left(\theta_{p}\right.$ is the theta function for $J\left(W_{p}\right)$ and $\theta_{p-1}$ is the theta function for $A_{p-1}$.) Consequently any homogeneous theta relation holding for $A_{p, 1}$ becomes a relation for $J\left(W_{p}\right)$ by replacing

$$
\theta_{p-1}\left[\begin{array}{c}
\varepsilon \\
\varepsilon^{\prime}
\end{array}\right]
$$

by

$$
\sqrt{\theta_{p}\left[\begin{array}{cc}
0 & \varepsilon \\
0 & \varepsilon^{\prime}
\end{array}\right] \theta_{p}\left[\begin{array}{cc}
0 & \varepsilon \\
1 & \varepsilon^{\prime}
\end{array}\right]}
$$

For genus four a special theta relation is of type $\sqrt{r_{1}}+\sqrt{r_{2}}+\sqrt{r_{3}}=0$ where each $r_{k}$ is a product of eight thetanulls. Thus a very special theta relation for genus five is of the form $\sqrt[4]{S_{1}}+\sqrt[4]{S_{2}}+\sqrt[4]{S_{3}}=0$ where each $S_{i}$ is a product of sixteen thetanulls. For genus six the corresponding very special relation will have four terms; for genus seven, six.

Since generic Riemann surfaces of genus 4, 5, and 6 are all 4-sheeted coverings of $\mathbf{P}_{1}$, very special relations describe the trigonal locus in Teichmüller space for genus 5 , 6 , and 7, at least locally. In particular, for genus 5 one such relation will describe the trigonal locus, which has codimension one in Teichmüller space for genus 5 . 


\section{REFERENCES}

1. R. D. M. Accola, Strongly branched coverings of closed Riemann surfaces, Proc. Amer. Math. Soc. 26 (1970), 315-322.

2. Riemann surfaces, theta functions, and abelian automorphism groups, Lecture Notes in Math., vol. 483, Springer-Verlag, Berlin and New York, 1975.

3. Plane models for Riemann surfaces admitting certain half-canonical linear series. II, Trans. Amer. Math. Soc. 263 (1981), 243-259.

4. A. Andreotti and A. L. Mayer, On period relations for abelian integrals on algebraic curves, Ann. Sculoa Norm. Sup. Pisa (3) 21 (1967), 189-238.

5. G. Castelnuovo, Ricerche di geometria sulle curve algebriche, Atti Accad. Sci. Torino 24 (1889) ( Memorie Scelte, Zanichelli, Bologna, 1937, p. 19).

6. J. L. Coolidge, Algebraic plane curves, Oxford, 1931; reprint, Dover, New York.

7. D. Eisenbud and J. Harris, Curves in projective space, Les Presses de l'Univ. de Montreal, 1982, Theorem 3.15.

8. H. M. Farkas and I. Kra, Riemann surfaces, Springer-Verlag, Berlin and New York, 1980.

9. P. Griffiths and J. Harris, Principles of algebraic geometry, Wiley, New York, 1978.

10. A. Krazer, Lehrbuch der Thetafunktionen, Teubner, Leipzig, 1903; reprint, Chelsea, New York.

11. H. H. Martens, On the varieties of special divisors on a curve. II, J. Reine Angew. Math. 233 (1968), $89-100$.

12. D. Mumford, Curves and their Jacobians, Univ. of Michigan Press, 1975.

13. H. E. Rauch and H. M. Farkas, Theta functions with applications to Riemann surfaces, Williams and Wilkin, 1974.

14. S. Recillas, Jacobians of curves with $g_{4}^{1}$ 's are the Prym's of trigonal curves, Bol. Soc. Mat. Mexicana (2) 19 (1974), 9-13.

15. F. Severi, Vorlesunger über algebraische Geometric, Teubner, Leipzig, 1921; reprint, Johnson, reprint, New York.

16. J. Thomae, Beitrag zur Bestimmung von $\theta(0,0, \ldots, 0)$ durch die Klassmoduln algebraischer Functionen, J. Reine Angew. Math. 71 (1870), 201-222.

17. R. J. Walker, Algebraic curves, Princeton, 1950; reprint, Dover, New York.

Department of Mathematics, Brown University, Providence, Rhode Island 02912 